\title{
Interactions in Atomic and Ionic Liquids
}

\author{
Jean-Louis Bretonnet \\ LCP-A2MC (EA 3469), University of Lorraine, Metz, France
}

\section{Email address:}

jean-louis.bretonnet@univ-lorraine.fr

\section{To cite this article:}

Jean-Louis Bretonnet. Interactions in Atomic and Ionic Liquids. Advances in Materials. Vol. 9, No. 4, 2020, pp. 68-93. doi: 10.11648/j.am.20200904.12

Received: February 3, 2020; Accepted: July 9, 2020; Published: November 21, 2020

\begin{abstract}
This review seeks to describe, from first principles, the nature of the interaction forces in atomic and ionic liquids. The atoms and molecules made up of dipoles and multipoles interact with van der Waals forces, while the ionic systems are viewed as pseudoions interacting through effective forces depending on the electronic structure and the physical ionic arrangement. The interplay between these two aspects of materials is quite complex and forms the main subject of this review. As it will be shown, the two-component system of interacting electrons and ions can be reduced, in second order perturbation theory, to an effective one-component system made up of pseudoions acting under the influence of two-body, central, screened potentials. These potentials result from a weak interaction between the electrons and the ions, deduced from the pseudopotential theory. Once the interatomic forces are known, the atomic structure and the electronic transport properties can be determined by methods of classical mechanics and quantum mechanics. Besides, a large volume-dependent term in the free energy, independent of the ionic positions, which distinguishes the conducting liquids from the simple isolator liquids like argon, is indispensable for explaining the thermodynamical properties.
\end{abstract}

Keywords: T-X Family, Exponentiated Exponential Distribution, Order Statistics, Shannon Entropy and Likelihood Ratio Test

\section{Classification of Liquids}

A liquid is a phase of condensed matter in which the density is further removed from that of the gas phase than that of the corresponding solid phase. Because of this large difference of density between liquids and gases, the thermodynamic functions of liquids cannot be developed as a function of the density, as done for the gases. But, although the density of liquids is close to that of solids, at a similar temperature, the lack of translational symmetry and long-range order does not allow a theoretical description of liquids as easily as for solids.

Liquids have been classified for a long time according to their electronic or atomic transport properties. For example, the liquids (like solids) can be divided into insulators and conductors; for conductors, it must be still indicated whether the conduction is ionic (the conductivity of molten salts varies from 1 to $10^{3} \Omega^{-1} \cdot \mathrm{m}^{-1}$ ) or electronic (the conductivity of liquid metals is of the order of $10^{6} \Omega^{-1} \cdot \mathrm{m}^{-1}$ ). However, the liquids can also be classified according to their ability to flow more or less easily. While most of simple liquids have low dynamic viscosities $\left(10^{-3} \mathrm{~Pa} . \mathrm{s}\right)$, certain organic fluids may have viscosities much larger (1 Pa.s).
Among the possible classifications of liquids, the least controversial is the one made on the basis of the interactions acting between atoms or molecules, because a specific interaction dominates in each type of liquid. This review is solely devoted to the simple liquids, that is to say to liquids characterized by interactions in spherical symmetry whose forces have the center of mass of the particles as the application point. For such systems, the potential energy $U$ is written as a sum of $N$-body potentials:

$$
\begin{aligned}
U_{N}\left(\mathbf{r}_{1}, \ldots, \mathbf{r}_{N}\right) & =\sum_{i<j} u_{2}\left(\mathbf{r}_{i}, \mathbf{r}_{j}\right)+\sum_{i<j<k} u_{3}\left(\mathbf{r}_{i}, \mathbf{r}_{j}, \mathbf{r}_{k}\right) \\
& +\sum_{i<j<k<l} u_{4}\left(\mathbf{r}_{i}, \mathbf{r}_{j}, \mathbf{r}_{k}, \mathbf{r}_{l}\right)+\ldots
\end{aligned}
$$

where $u_{2}\left(\mathbf{r}_{i}, \mathbf{r}_{j}\right)$ is the 2-body potential, $u_{3}\left(\mathbf{r}_{i}, \mathbf{r}_{j}, \mathbf{r}_{k}\right)$ the 3 -body potential, etc. This notation clearly indicates that if three particles $(i, j, k)$ are interacting, their potential energy consists of the sum of the 2-body potential $\left[u_{2}\left(\mathbf{r}_{i}, \mathbf{r}_{j}\right)+u_{2}\left(\mathbf{r}_{j}, \mathbf{r}_{k}\right)+u_{2}\left(\mathbf{r}_{k}, \mathbf{r}_{i}\right)\right]$ plus the 3-body potential $u_{3}\left(\mathbf{r}_{i}, \mathbf{r}_{j}, \mathbf{r}_{k}\right)$.

The most representative simple liquids are the noble gases: 
argon, krypton, xenon as classic liquids, as well as neon and helium, which are considered as quantum liquids when the de Broglie wavelength of the atoms is of the same order of magnitude as the interatomic distance. At low densities, the methane $\left(\mathrm{CH}_{4}\right)$ can also be regarded as a simple liquid because it consists of nearly spherical molecules.

In the category of simple liquids, enter also, without restriction, the liquid metals such as alkali metals ( $\mathrm{Na}, \mathrm{K}, \mathrm{Cs}$ ...), noble metals ( $\mathrm{Ag}, \mathrm{Au}, \mathrm{Cu}$ ) and most of polyvalent metals. Germanium and silicon can also be seen as such, although they have a pronounced tendency to form covalent bonds. Unlike the noble gas atoms, the metal atoms have incomplete electron shells occupied by the valence electrons forming a gas of nearly free electrons. Thus, it may seem strange that a metal, composed of ions immersed in an electron gas, can be regarded as a simple liquid. In fact, this is legitimate to the extent that the metal can be thought of as a set of pseudoions interacting with a pseudopotential, the concept of which was developed by Phillips and Kleinman[1]. Although the concept of pseudopotential is more difficult to justify for the transition metals and rare earth metals than for the normal metals (alkali and polyvalent) - due to the hybridization effects between the valence electrons and core electrons - the fact remains that, from a structural point of view, all the liquid metals are considered as simple liquids. It should be mentioned that the pseudopotential formalism is a powerful tool used to define the pair potential in liquid metals, which depends on the density and temperature, unlike the pair potential of the rare gases.

Binary alloys and liquids having important directional effects will not be investigated here. Examples of these liquids are the homonuclear $\left(\mathrm{N}_{2}, \mathrm{O}_{2}, \mathrm{H}_{2}\right)$ and heteronuclear (CO) molecular liquids, the associated liquids (water, glycerol ...) characterized by hydrogen bonds and the polar liquids $\left(\mathrm{HBr}, \mathrm{SO}_{2} \ldots\right)$ whose molecules have a strong asymmetry or pronounced electrostatic interactions.

Another class of simple liquids, with a structural length scale between 10 nanometers and one micron $(1000 \mathrm{~nm})$, will not be investigated either. These systems are composed of mesoscopic particles 1000 times larger than atoms, immersed in an aqueous solution. They include the colloidal suspensions, spherical polymers, biological macromolecules, etc. Although they are more sensitive to mechanical stresses than the atomic systems, their thermodynamic and structural properties can be calculated with the same theoretical models as those of the atomic systems. Their interparticle potentials will be examined in another paper.

\section{Typology of Microscopic Interactions}

\subsection{Interactions at Short and Long Distances}

The low compressibility and the relatively high density of liquids show respectively the existence of repulsive forces at short distances and attractive forces at long distances. The repulsive forces prevent the collapse of the matter on itself and the attractive forces give the cohesive properties. Generally the interactions between two entities (molecules or atoms) are investigated in terms of potential energy $u_{2}(r)$ and not in terms of force $f(r)\left(=-\frac{d u_{2}(r)}{d r}\right)$. The potential energy (potential) is calculated using quantum mechanics.

The potential associated with the repulsive forces between two atoms not overlapping, has usually the form:

$$
u_{2}^{r e p}(r)=\sum_{i} P_{i}(r) \exp \left(-\frac{r}{r_{c_{i}}}\right),
$$

where $P_{i}(r)$ are polynomials in $r$ and $r_{c_{i}}$ parameters depending on the individual atoms. This interaction potential takes into account the electrostatic repulsion exerted between the two nuclei and the effects due to the Pauli exclusion principle. In practice, simplified forms of $u_{2}^{r e p}(r)$ are used such as that of Born and Mayer[2]:

$$
u_{2}^{r e p}(r)=a \exp \left(-\frac{r}{b}\right)
$$

where $a$ and $b$ are two constants determined by adjusting certain physical properties of the experimental results. But the value of $b$ can also be estimated with good accuracy by the relation $b \simeq\left(\frac{a_{0}}{\sqrt{2}}\right) Z^{1 / 3}$, where $a_{0}(=0,0529 \mathrm{~nm})$ is the Bohr radius and $Z$ the atomic number. Sometimes the following formula, in inverse power of $r$, is used:

$$
u_{2}^{r e p}(r)=\frac{c}{r^{m}},
$$

where $c$ and $m$ are two constants to be determined.

Regarding the potentials associated with the attractive forces at long range, they contain various contributions that are better known than those of the repulsive potential. These attractive forces, discovered by London but called the van der Waals forces, result from the presence of dipoles or multipoles in molecules and atoms.

\subsection{Interaction Between a Dipole and a Point Charge}

In this subsection we recall the calculation of the potential energy of interaction between a dipole and a point charge (monopole), which is at the basis of the study of the van der Waals forces. An electrostatic dipole consists of a pair of two point charges of opposite signs, $+q$ and $-q$, located at points $A$ and $B$. The electric dipole moment is defined by the vector p:

$$
\mathbf{p}=q . \mathbf{B A}
$$

To calculate the potential energy of interaction between the dipole and a point charge $Q$, we fix the point $O$ in the middle of $A B=l$ and assume that the charge $Q$ is located at the distance $O M=r$ (with $r \gg l$ ) in the direction $\theta$ (Figure 1). 


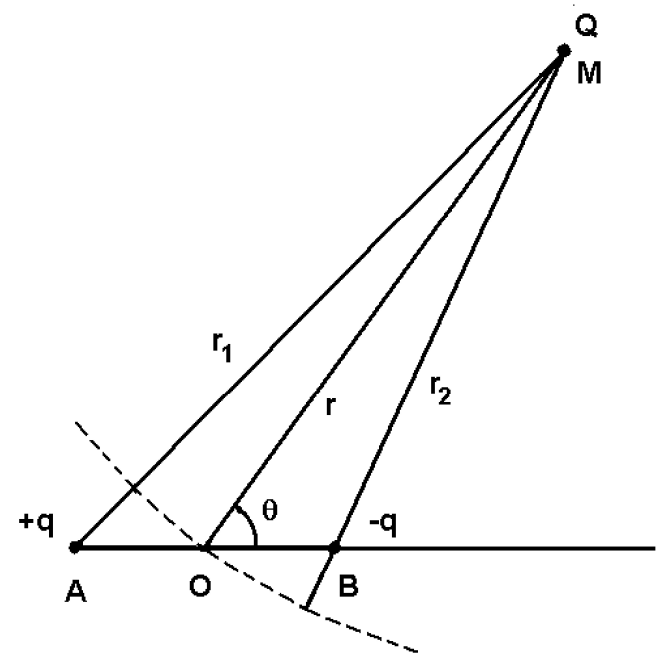

Figure 1. Schematic representation of the dipole $A B$ interacting with the point charge $Q$.

The potential energy is given by Coulomb's law:

$$
u(r)=+\frac{q Q}{4 \pi \varepsilon_{0} r_{1}}-\frac{q Q}{4 \pi \varepsilon_{0} r_{2}}=\frac{q Q}{4 \pi \varepsilon_{0}}\left(\frac{r_{2}-r_{1}}{r_{1} r_{2}}\right),
$$

where:

$$
\begin{aligned}
& r_{1}=A M \simeq r+\frac{l}{2} \cos \theta \\
& r_{2}=B M \simeq r-\frac{l}{2} \cos \theta
\end{aligned}
$$

$$
\frac{r_{2}-r_{1}}{r_{1} r_{2}}=\frac{-l \cos \theta}{r^{2}-\frac{l^{2}}{4} \cos ^{2} \theta} \simeq-\frac{l \cos \theta}{r^{2}} .
$$

In substituting this result in equation (6), and taking into account of equation (5), we are in position to write the potential energy $u(r)$ in the form:

$$
u(r)=-\frac{q Q}{4 \pi \varepsilon_{0}} \frac{l \cos \theta}{r^{2}}=-\frac{Q p}{4 \pi \varepsilon_{0}} \frac{\cos \theta}{r^{2}} .
$$

It should be noted, in passing, that the electric field created by the dipole at the point $M$ is $\mathbf{E}=-\operatorname{grad} V$, where $V$ is the electrostatic potential associated with the potential energy $u(r)$ by the relation $u(r)=Q . V(r)$. Therefore, the electric field modulus at the point $M$ is expressed as:

$$
E(r)=\frac{1}{Q} \frac{d u(r)}{d r}=\frac{p \cos \theta}{4 \pi \varepsilon_{0}} \frac{2}{r^{3}} .
$$

so that:

\subsection{Intermolecular Interactions}

Often, the potential energy is calculated between a set of charges $q_{i}$, gathered in a small volume, and a point charge $Q$ located at a distance $r$ of this volume.

If the sum of charges in the volume is not zero $\left(\sum q_{i} \neq 0\right.$, as in an ion), it can be taken that the total charge $\sum q_{i}$ is concentrated at the centroid, so that the potential energy is calculated with the formula:

$$
u(r)=\frac{1}{4 \pi \varepsilon_{0}} \frac{Q \sum q_{i}}{r} .
$$

But to get better accuracy, the calculation has to be performed for all the individual charges, which amounts to evaluating the potential energy $u(r)$ as an expansion in powers of $1 / r$.

If the sum of charges in the volume is zero $\left(\sum q_{i}=0\right.$, as in a molecule), the first term of the series in $1 / r$ is zero. In addition, if all the charges $q_{i}$ are reduced to a dipole (consisting of one charge $-q$ and one charge $+q$ ), the potential energy between the point charge $Q$ and this dipole is given by equation (10). It is proportional to $1 / r^{2}$. But it may happen that the charges are reduced, not to a dipole, but to a quadrupole (set of 4 charges $-q,+q,-q^{\prime},+q^{\prime}$ arranged symmetrically about the centroid). In this case, the term $1 / r^{2}$ is zero and the potential energy between the point charge $Q$ and the quadrupole is proportional to $1 / r^{3}$. If there is no quadrupole, the term $1 / r^{3}$ is nonexistent and an octupole (set of 8 charges arranged symmetrically around the centroid) must be considered, and so on. Thus, a set of charges can be decomposed into multipoles (dipoles, quadrupoles, octupoles...) playing a significant role in certain circumstances.

The simplest polar systems are formed of monopoles. These systems, such as molten salts, consist of charges $Q=z e$ (e.g. $\mathrm{NaCl}$ composed of charges $+e$ and $-e$ ). Other polar systems are made up of permanent dipoles (hydrogen bromide composed of uncharged molecules with a dipole moment $p_{d}$ ) or still of permanent quadrupoles (liquid nitrogen composed of molecules having a quadrupole moment $p_{q}$ ). The potential energies between these various entities are calculated by direct application of the laws of electrostatics. For example, the potential energy between one point charge $Q$ and one dipole moment $p_{d}$ is given by equation (10), i.e.:

$$
u_{Q d}(r)=-\frac{Q p_{d}}{4 \pi \varepsilon_{0}} \frac{\cos \theta}{r^{2}} .
$$

Similarly, the potential energy between one point charge $Q$ and one quadrupole moment $p_{q}$ is calculated with the formula:

$$
u_{Q q}(r)=-\frac{Q p_{q}}{16 \pi \varepsilon_{0} r^{3}}\left(3 \cos ^{2} \theta-1\right) .
$$

In the case of the potential energy between two dipoles of dipole moments $p_{d 1}$ and $p_{d 2}$, it is expressed as:

$$
u_{d d}(r)=-\frac{p_{d 1} \cdot p_{d 2}}{4 \pi \varepsilon_{0} r^{3}}\left[2 \cos \theta_{1} \cos \theta_{2}-\sin \theta_{1} \sin \theta_{2} \cos \left(\phi_{1}-\phi_{2}\right)\right] .
$$

Thus, it is possible to calculate the potential energies between all the entities: monopole-monopole $(Q Q)$, monopole-dipole $(Q d)$, monopole-quadrupole $(Q q)$, monopole-octupole $(Q o) \ldots$, dipole-dipole $(d d)$, dipole-quadrupole $(d q) \ldots$, 
quadrupole-quadrupole $(q q)$... and so on. All these expressions are given for specific position and orientation of the entities. As an example, figure 2 .

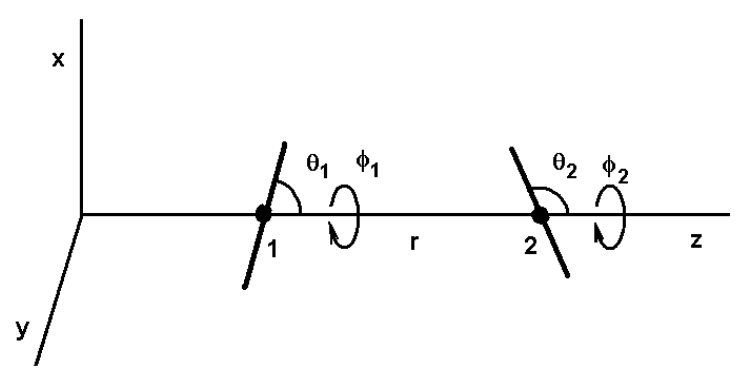

Figure 2. Positions and orientations of two dipoles separated by $r$ along the $z$ axis. The orientations are defined by the angles $\theta_{1}, \phi_{1}, \theta_{2}$ and $\phi_{2}$.

shows two dipoles separated by $r$ on the $z$ axis, where $\theta_{1}$ and $\theta_{2}$ are the angles between the dipoles and the $z$ axis, and $\phi_{1}$ and $\phi_{2}$ are the azimuth angles. The five parameters $r, \theta_{1}, \theta_{2}, \phi_{1}$ and $\phi_{2}$ completely determine the positions of the two dipoles and the potential energy $u_{d d}\left(r, \theta_{1}, \theta_{2}, \phi_{1}, \phi_{2}\right)$.

Keesom[3] suggested calculate the thermodynamic properties of these systems with the average potential energy $\bar{u}(r, T)$, obtained by integrating $u(r)$ over all orientations of the molecules according to the relation:

$$
\bar{u}(r, T)=\frac{\iint u(r) \exp [-\beta u(r)] \sin \theta_{1} d \theta_{1} d \phi_{1} \sin \theta_{2} d \theta_{2} d \phi_{2}}{\iint \exp [-\beta u(r)] \sin \theta_{1} d \theta_{1} d \phi_{1} \sin \theta_{2} d \theta_{2} d \phi_{2}},
$$

where the Boltzmann factor $\exp (-\beta u)$ is used, with $\beta=\frac{1}{k_{B} T}$, in order to give a greater weight to the orientations of molecules corresponding to states of lowest energy. As a result, the average potential energy $\bar{u}(r, T)$ loses its angular dependence, but acquires a temperature dependence. Since these integrals are very difficult to calculate, the Taylor expansion of the exponential function is generally used around the average value of the potential energy, i.e.:

$$
\bar{u}(r, T)=\frac{\iint u \cdot[1-\beta(u-\bar{u})+\ldots] \sin \theta_{1} d \theta_{1} d \phi_{1} \sin \theta_{2} d \theta_{2} d \phi_{2}}{\iint[1-\beta(u-\bar{u})+\ldots] \sin \theta_{1} d \theta_{1} d \phi_{1} \sin \theta_{2} d \theta_{2} d \phi_{2}} .
$$

This expression allows the evaluation of $\bar{u}(r, T)$ by the equation [4]:

$$
\begin{aligned}
\bar{u}(r, T) & =\frac{1}{64 \pi^{6}} \iint u \sin \theta_{1} d \theta_{1} d \phi_{1} \sin \theta_{2} d \theta_{2} d \phi_{2}-\frac{1}{k_{B} T} \frac{1}{64 \pi^{6}} \iint u^{2} \sin \theta_{1} d \theta_{1} d \phi_{1} \sin \theta_{2} d \theta_{2} d \phi_{2} \\
& -\frac{1}{k_{B} T}\left[\frac{1}{64 \pi^{6}} \iint u \sin \theta_{1} d \theta_{1} d \phi_{1} \sin \theta_{2} d \theta_{2} d \phi_{2}\right]^{2}+\ldots
\end{aligned}
$$

Below the expressions are shown for the average potential energies of the main molecular entities (monopole-monopole $Q Q$, monopole-dipole $Q d$, monopole-quadrupole $Q q$ and dipole-dipole $d d$ ):

$$
\begin{aligned}
\bar{u}_{Q Q}(r) & =\frac{Q^{2}}{r}, \\
\bar{u}_{Q d}(r) & =-\frac{1}{3 k_{B} T} \frac{Q^{2} p_{d}^{2}}{r^{4}}, \\
\bar{u}_{Q q}(r) & =-\frac{1}{20 k_{B} T} \frac{Q^{2} p_{q}^{2}}{r^{6}}, \\
\bar{u}_{d d}(r) & =-\frac{2}{3 k_{B} T} \frac{p_{d 1}^{2} \cdot p_{d 2}^{2}}{r^{6}} .
\end{aligned}
$$

It is found that the greater the number of poles in a molecule, the faster the potential energy drops away with distance. Thus, the potential energy between monopoles varies like $r^{-1}$, the dipole-monopole potential energy like $r^{-4}$, the dipole- dipole potential energy like $r^{-6}$. It is also demonstrated that the dipole-quadrupole potential energy varies like $r^{-8}$, the quadrupole-quadrupole potential energy like $r^{-10}$, etc.

Sometimes a set of charges having no permanent dipole moment acquires an electric dipole moment when placed in an electric field. Indeed, it can be understood that a molecule composed of electrons and nuclei with no permanent dipole moment can, under the action of an external electric field, see the electrons and nuclei move in opposite directions, creating a new equilibrium configuration with an induced dipole moment. Naturally, superimposing permanent dipole moments on induced dipole moments is possible. In this case, the potential energy is composed of two contributions. One is due to the interaction between permanent dipoles and the other to the interaction between induced dipoles.

Usually, the purely electrostatic forces between polar molecules (permanent dipoles) are weaker than those acting 
between nonpolar molecules (induced dipoles), and might be therefore neglected. By contrast, in highly polar molecules, such as those of water or acetone, the electrostatic forces are masked by strong chemical interactions that depend on the orientation. These will not be discussed here.

\subsection{Interatomic Interactions}

The electrostatic forces, which have been described in the case of spherical molecules (nonpolar), gain a special importance in the case of atoms. The reason is that the fluctuations of electron density in an atom produces an instantaneous dipole.

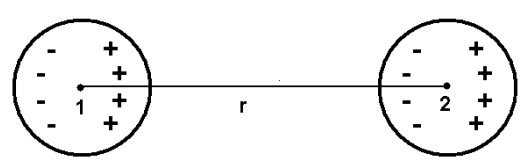

Figure 3. Interaction between the induced dipoles created in two neighboring atoms.

Indeed, the movement of the electrons around the nucleus moves the centroid of negative charges, and contributes to the formation of an instantaneous dipole that fluctuates in magnitude and direction at the rotation frequency of the electrons $\left(\sim 10^{15} \mathrm{~s}^{-1}\right)$. In addition, the electric field created by the instantaneous dipole affects the movement of electrons in a neighboring atom giving rise to an induced dipole, which fluctuates in phase with the first dipole. Generally, the two dipoles are aligned and exert one upon another a force of attraction. This force is called the dispersion force for the following reason. The electric field created by the instantaneous dipole of the first atom propagates at the speed of light towards the second atom, and the dipole induced in the second atom in turn emits an electric field in direction to the first atom. If the two atoms are close enough, the two dipoles fluctuate in phase and their directions are identical. But if the atoms are far apart from each other, a phase shift occurs between the electric fields, hence a disorientation of the dipoles and a decrease in the strength of interaction. This effect is comparable to the dispersion of light in a medium of given refractive index.

The calculation of dispersion forces was made by London[5] with the perturbation theory of quantum mechanics, but the general expression can be established simply[6] using the model of the Bohr atom ${ }^{1}$. Considering the Bohr atom, it

${ }^{1}$ The energy of the Bohr atom in the ground state is:

$$
E_{0}=-\frac{m e^{4}}{8 \varepsilon_{0}^{2} h^{2}}=-2.18 .10^{-18} \mathrm{~J} \quad(\text { i.e. } 13.6 \mathrm{eV}),
$$

with:

$$
\begin{aligned}
m & =0.911 \cdot 10^{-30} \mathrm{~kg}, \\
e & =1.602 \cdot 10^{-19} \mathrm{C}, \\
\varepsilon_{0} & =8.854 \cdot 10^{-12} \mathrm{~J}^{-1} \cdot \mathrm{C}^{2} \cdot \mathrm{m}^{-1}, \\
h & =6 \cdot 622 \cdot 10^{-34} \mathrm{~J} . \mathrm{s} .
\end{aligned}
$$

can be assumed that the instantaneous dipole moment, created between the nucleus and the electron, is roughly speaking (Eq. 5):

$$
p_{1} \simeq e a_{0},
$$

where $e$ is the electron charge and $a_{0}$ the Bohr radius:

$$
a_{0}=\frac{e^{2}}{8 \pi \varepsilon_{0} h \nu} .
$$

The energy of the Bohr atom in its ground state, also called the ionization potential, is $I=h \nu$, where $\nu\left(=3.3 .10^{15} \mathrm{~s}^{-1}\right)$ is the characteristic frequency associated with the movement of the electron in its orbit.

The electric field produced by an instantaneous dipole at distance $r$ from the atom (Eq. 11) is on average:

$$
E(r) \simeq \frac{p_{1}}{4 \pi \varepsilon_{0} r^{3}}=\frac{e a_{0}}{4 \pi \varepsilon_{0} r^{3}}
$$

However, a second neutral atom situated in this electric field, at distance $r$, undergoes a polarization and acquires an induced dipole moment:

$$
p_{2}=\alpha E(r)=\frac{\alpha e a_{0}}{4 \pi \varepsilon_{0} r^{3}}
$$

where $\alpha$ is the polarizability of this atom. This quantity, which measures the ability to move the electronic distribution in the atom, is proportional to the volume of the atom:

$$
\alpha \simeq 4 \pi \varepsilon_{0} a_{0}^{3} .
$$

Thus the potential energy of attraction between the dipoles $p_{1}$ and $p_{2}$ is obtained by performing the product of the dipole moment of the second atom (Eq. 25) by the electric field, on this spot, created by the first atom (Eq. 24), namely:

$$
\begin{aligned}
u_{d d}^{d i s p}(r) & =-E(r) p_{2}, \\
& =-\frac{\alpha e^{2} a_{0}^{2}}{\left(4 \pi \varepsilon_{0}\right)^{2} r^{6}} .
\end{aligned}
$$

Therefore, using equations (23) and (26), the potential energy between two atoms separated by distance $r$ is written as:

$$
u_{2}^{d i s p}(r)=-\frac{C_{6}}{r^{6}}
$$

where the constant $C_{6} \simeq \frac{2 \alpha^{2} h \nu}{\left(4 \pi \varepsilon_{0}\right)^{2}}$ depends on the square of the polarizability $\alpha$ and the ionization potential $h \nu$, as

But $E_{0}(=-h \nu)$ also corresponds to the energy of the electron on its lowest orbit, of radius:

$$
a_{0}=\frac{\varepsilon_{0} h^{2}}{\pi m e^{2}}=0.0529 .10^{-9} \mathrm{~m} .
$$

Then, the characteristic frequency associated with the movement of the electron on its orbit is given by the expression:

$$
\nu=-\frac{E_{0}}{h}=\frac{m e^{4}}{8 \varepsilon_{0}^{2} h^{3}}=\frac{1}{a_{0}} \frac{e^{2}}{8 \pi \varepsilon_{0} h}=3.3 .10^{15} \mathrm{~s}^{-1},
$$

which links $a_{0}$ with $\nu$. 
could be demonstrated by a precise calculation using quantum mechanics.

In dense systems, the presence of a third atom brings a further contribution to the two-body potential (Eq. 28). This is a three-body potential calculated by taking into account the dispersion forces between three dipoles[7, 8]. The analytical expression of the triple dipole potential, varying as $r^{-9}$, is expressed as a function of the atomic polarizability $\alpha$ and the constant $C_{6}$ as:

$$
u_{d d d}^{d i s p}\left(r_{12}, r_{23}, r_{13}\right)=\frac{3}{4} \alpha C_{6}\left[\frac{1+3 \cos \theta_{1} \cos \theta_{2} \cos \theta_{3}}{r_{12}^{3} r_{23}^{3} r_{13}^{3}}\right]
$$

where the angles $\theta_{1}, \theta_{2}$ and $\theta_{3}$ are those of the triangle formed by three atoms $(1,2,3)$ with sides $r_{12}=\left|\mathbf{r}_{2}-\mathbf{r}_{1}\right|, r_{23}=$ $\left|\mathbf{r}_{3}-\mathbf{r}_{2}\right|$ and $r_{13}=\left|\mathbf{r}_{3}-\mathbf{r}_{1}\right|$.

It should be mentioned that the full calculations of the two-body potential $u_{2}^{d i s p}(r)$ and three-body potential $u_{3}^{d i s p}\left(r_{12}, r_{23}, r_{13}\right)$ allow us to generalize equations (28) and (29), respectively in the following forms:

$$
u_{2}^{d i s p}(r)=-\frac{C_{6}}{r^{6}}-\frac{C_{8}}{r^{8}}-\frac{C_{10}}{r^{10}}+\ldots,
$$

and

$$
u_{3}^{d i s p}=u_{d d d}^{d i s p}+u_{d d q}^{d i s p}+u_{d q q}^{d i s p}+u_{q q q}^{d i s p}+\ldots
$$

The various terms of these expressions have been calculated by the perturbation theory to fourth order, applied to the electronic structure of atoms. The details of these issues will be found in specialized articles[9, 10, 11].

\subsection{Potentials in Liquid Rare Gases}

Although the coefficients $C_{6}, C_{8}, C_{10}$ in equation (30) are related to the energies of atoms, their values are only approximate. For example, for argon, they are:

$$
\begin{aligned}
C_{6} & \simeq \frac{3 \alpha^{2} I}{4} \\
C_{8} & \simeq 5 C_{6}\left(\frac{I}{f e^{2}}\right), \\
C_{10} & \simeq 30 C_{6}\left(\frac{I}{f e^{2}}\right)^{2} .
\end{aligned}
$$

With a value of the atomic polarizability $\alpha=1.64 .10^{-3}$ $\mathrm{nm}^{3}$ and an ionization potential of $I=17.5 \mathrm{eV}$, the calculated value of $C_{6}$ is worth about $9 / 10$ of the experimental value. The values of the coefficients $C_{i}$ are only approximate and the attractive potential in liquid rare gases are not rigorously defined. It is a matter of fact that the calculation of the repulsive potential is even more questionable. This explains why the construction of potentials in liquid rare gases is a combination of ab-initio and empirical calculations.

The first explicit calculation of the interaction between two helium atoms has been done by Slater and Kirkwood[12]. The resulting expression is the sum of a decay exponential function for the repulsive contribution and a power function varying as $r^{-6}$ for the attractive contribution:

$$
u(r)=a \exp \left(-\frac{r}{b}\right)-\frac{C_{6}}{r^{6}},
$$

where $a, b$ and $C_{6}$ are three constants. It is found that the repulsive interaction is of the Born-Mayer type (Eq. 3) and the attractive interaction is due to induced dipole-induced dipole dispersion forces (Eq. 28).

One of the most realistic two-body potentials for noble gases has been proposed by Aziz and Slaman[13] under the form:

$$
u_{2}(x)=A \exp \left(-\alpha x+\beta x^{2}\right)-F(x) \sum_{i=0}^{2} \frac{C_{2 i+6}}{x^{2 i+6}},
$$

where $x=r / r_{m}$ is the reduced distance and $r_{m}$ the position of the potential well. To eliminate the divergence introduced by the terms of dispersion to small distances, the authors have chosen to use the amortized function $F(x)$ :

$$
F(x)= \begin{cases}\exp \left[-\left(\frac{D}{x}-1\right)^{2}\right] & \text { if } x<D \\ 1 & \text { if } x \geq D .\end{cases}
$$

This potential model is composed of eight parameters determined by fitting some experimental quantities, the values of which will be found in the original article, for various rare gases. It should be noted that this potential is a pair potential and that the three-body Axilrod-Teller potential have to be added to improve the accuracy of calculation of the physical properties.

\section{Law of Corresponding States}

\subsection{Empirical Potential}

To facilitate the study of properties of liquids, it is desirable to use simpler expressions of the potential, although realistic, in order to simplify the analytical calculations. For example, the Gaussian potential is sometimes used to represent the repulsive part at short distance, because it facilitates the calculation of integrals that contain it.

As a realistic potential, the square-well potential:

$$
u(r)=\left\{\begin{array}{cl}
+\infty & \text { if } r \leq \sigma \\
-\varepsilon & \text { if } \sigma<r \leq \lambda \alpha \\
0 & \text { if } r>\lambda \alpha
\end{array}\right.
$$

is widely used for its simple mathematical form. It correctly predicts the thermodynamic properties and the local structure of liquids. Without the square well, this potential reduces to the hard-sphere potential, which is also widely used.

There are a plethora of empirical potentials, but one that seems most appropriate to investigate the simple liquids is the Lennard-Jones potential. It is representative of many real potentials, insofar as it can describe numerous physical properties, without undue complexity of the calculations. Its general form is: 


$$
u(r)=4 \varepsilon\left[\left(\frac{\sigma}{r}\right)^{m}-\left(\frac{\sigma}{r}\right)^{n}\right],
$$

where $\varepsilon$ and $\sigma$ are two parameters to be determined. The most commonly-used expression is that with $n=6$ and $m=2 n=12$. With a repulsive part varying as $r^{-12}$ and an attractive part as $r^{-6}$, it owns strong physical justification in view of equations (4) and (28). Moreover, the choice of the parametrization is particularly suited to the mathematical description of the function $u(r)$. Indeed, $\sigma$ is the value of $r$ that makes the potential zero and $\varepsilon$ is the depth of the potential well. By calculating the derivative $\frac{d u(r)}{d r}$, it is easy to see that the minimum position of the potential well is located at $r_{m}=$ $2^{1 / 6} \sigma$, with $u\left(r_{m}\right)=-\varepsilon$. The values of the parameters $\sigma$ and $\varepsilon$ are determined by fitting some experimental properties. They also allow estimating the coefficient $C_{6}\left(=4 \varepsilon \sigma^{6}\right)$. For argon,

the value of $C_{6}$ resulting of the empirical values of $\varepsilon$ and $\sigma$ is almost two times larger than the theoretical result.

Despite its limitations, the empirical potential of LennardJones has a universal behavior favouring the study of many simple liquids, which differ from each other by the scale factors $\sigma_{i} / \sigma_{j}$ and $\varepsilon_{i} / \varepsilon_{j}$. Indeed, it is reasonable to assume that the potential $u(r)$ of each simple liquid can be represented in terms of a universal function $u^{*}(r)$ as:

$$
u(r)=\varepsilon u^{*}\left(\frac{r}{\sigma}\right)
$$

which is typical of the Lennard-Jones potential.

Note that the equation of state of liquids is expressed by means of the configuration integral $Z_{N}(V, T)$, according to the equation:

$$
p=k_{B} T \frac{\partial}{\partial V} \ln Z_{N}(V, T)
$$

Using the expression for $Z_{N}(V, T)$, the pressure is given by the following equation[14]:

$$
p=k_{B} T \frac{N}{V}-\frac{1}{3 V} \frac{1}{Z_{N}(V, T)} \sum_{i} \sum_{j>i} \iint_{3 N}\left[r_{i j} \frac{\partial u\left(r_{i j}\right)}{\partial r_{i j}}\right] \times \exp \left[-\beta U\left(\mathbf{r}^{N}\right)\right] d \mathbf{r}_{1} \ldots d \mathbf{r}_{N},
$$

where $U\left(\mathbf{r}^{N}\right)=\sum_{i} \sum_{j>i} u\left(r_{i j}\right)$ is the potential energy of liquid consisting of $N$ atoms. When the potential energy is assumed to be a sum of pair potentials of the universal type:

$$
U\left(\mathbf{r}^{N}\right)=\sum_{i} \sum_{j>i} u\left(r_{i j}\right)=\sum_{i} \sum_{j>i} \varepsilon u^{*}\left(\frac{r_{i j}}{\sigma}\right)
$$

the pressure may be written in the functional form:

$$
p=k_{B} T \frac{N}{V}-\frac{\varepsilon N}{3 V} g^{*}\left(\frac{\varepsilon}{k_{B} T}, \frac{N \sigma^{3}}{V}\right),
$$

where $g^{*}\left(\frac{\varepsilon}{k_{B} T}, \frac{N \sigma^{3}}{V}\right)$ is a dimensionless function depending on the nature of the potential $u^{*}$, i.e. the parameters $\varepsilon$ and $\sigma$. It follows that the pressure depends on the temperature $T$, the volume $V$ and the universal function $u^{*}$, through the function $g^{*}$. However, if the two parameters $\varepsilon$ and $\sigma$ are used to define the reduced variables:

$$
T^{*}=\frac{k_{B} T}{\varepsilon}, \quad V^{*}=\frac{V}{N \sigma^{3}}, \quad p^{*}=\frac{p \sigma^{3}}{\varepsilon},
$$

equation (43) takes the reduced form:

$$
\frac{p \sigma^{3}}{\varepsilon}=\frac{k_{B} T}{\varepsilon} \frac{N \sigma^{3}}{V}-\frac{N \sigma^{3}}{3 V} g^{*}\left(\frac{\varepsilon}{k_{B} T}, \frac{N \sigma^{3}}{V}\right),
$$

or:

$$
p^{*}\left(T^{*}, V^{*}\right)=\frac{T^{*}}{V^{*}}-\frac{1}{3 V^{*}} g^{*}\left(T^{*}, V^{*}\right) .
$$

This shows that the reduced pressure $p^{*}$ is a universal function of the reduced temperature $T^{*}$ and the reduced volume $V^{*}$, for all the liquids described by a potential of the form $u(r)=\varepsilon u^{*}\left(\frac{r}{\sigma}\right)$. Equation (45) is known as the law of corresponding states stipulating that all the liquids of the same family, interacting with the potential $u^{*}$, obey the same equation of state in terms of reduced variables. As this reduced equation of state must apply to all the thermodynamic states, notably to the critical state, it is required that the values of $p_{c}^{*}$, $T_{c}^{*}$ and $V_{c}^{*}$ are universal constants for all the liquids described by the potential $u^{*}$. For the Lennard-Jones potential (12-6), the approximate values are $p_{c}^{*} \simeq 0.28, T_{c}^{*} \simeq 1.3$ and $V_{c}^{*} \simeq 1.33$, so that the product is equal to $\frac{p_{c}^{*} V_{c}^{*}}{T_{c}^{*}} \simeq 0.28$. The experimental values of $\frac{p_{c}^{*} V_{c}^{*}}{T_{*}^{*}}$ found for simple liquids[4] (Ar, Kr, Xe, $\mathrm{N}_{2}$, $\mathrm{O}_{2}, \mathrm{CO}_{2}, \mathrm{CH}_{4} \ldots$ ) are about $0.290 \pm 0.005$, which yields an acceptable test of the theory.

\subsection{Relationship Between Potential and Limits of Phase Stability}

One of the successes of numerical simulation has been to establish a relationship between the features of the interatomic potential and the limits of stability of phase diagrams, thus clarifying the circumstances of the liquid-solid and liquidvapor transitions. It has been shown, in particular, that the hard-sphere potential is able to correctly describe the atomic structure of liquids and predict the liquid-solid transition[15, 16]. By contrast, it is unable to predict the liquid-vapor transition, which is due to the presence of attractive dispersion forces. More specifically, the simulation results have shown that the liquid-solid phase coexistence curves are governed by the repulsive part of potential, that is to say, by the steric hindrance of the atoms. This was already contained in the phenomenological theories of melting, like the Lindemann theory that predicts the melting of a solid when the average displacement of atoms, relatively to their equilibrium positions on the lattice, exceeds the atomic diameter of $10 \%$. In other words, a substance melts when its volume exceeds by $30 \%$ the 
volume at $0 \mathrm{~K}$.
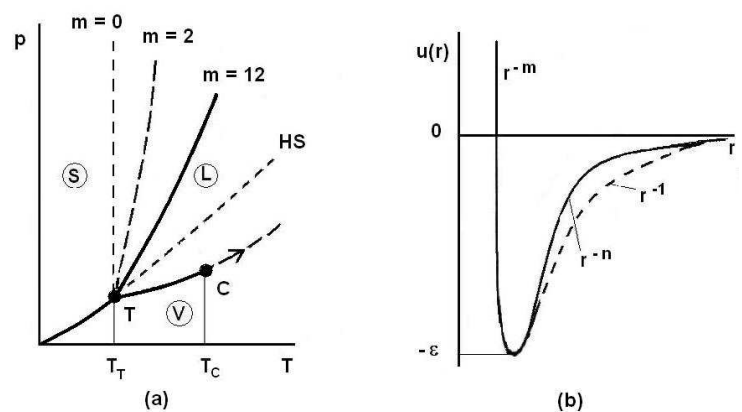

(b)

Figure 4. Schematic representations of the diagram $p(T)$ and the Lennard-Jones potential.

Figure 4 displays the diagram $p(T)$ of a pure substance as well as the Lennard-Jones potential (Eq. 38). One can see how the slope of the coexistence curve for solid-liquid phase varies with the repulsive part of the potential. Indeed, the greater the value of $m$, the steeper the repulsive part of the potential (Figure 4b) and the less sloping the solid-liquid coexistence curve (Figure 4a). Moreover, it becomes apparent that the Lennard-Jones potential predicts the liquid-vapor coexistence curve that begins at the triple point $\mathrm{T}$ and ends at the critical point C. A detailed analysis shows that the length of the segment TC is proportional to the depth of potential well $\varepsilon$. For rare gases, it is verified that $\left(T_{C}-T_{T}\right) k_{B} \simeq 0.55 \varepsilon$. It follows immediately from this relation that the liquid-vapor coexistence curve disappears when the potential well is absent $(\varepsilon=0)$.

In addition, the value of the slope of the segment TC also depends on the attractive part of the potential as shown by the Clausius-Clapeyron equation:

$$
\frac{d p}{d T}=\frac{L_{v a p}}{T_{v a p}\left(V_{v a p}-V_{l i q}\right)},
$$

where $L_{v a p}$ is the latent heat of vaporization at the corresponding temperature $T_{v a p}$ and $\left(V_{v a p}-V_{l i q}\right)$ the difference of specific volumes between vapor and liquid. To evaluate the slope $\frac{d p}{d T}$ of TC, at ambient pressure, we can estimate the ratio $\frac{L_{v a p}}{T_{v a p}}$ with Trouton's rule $\left(\frac{L_{v a p}}{T_{v a p}} \simeq 85\right.$ $\left.\mathrm{J} . \mathrm{K}^{-1} \cdot \mathrm{mol}^{-1}\right)$ and the difference of volumes $\left(V_{\text {vap }}-V_{l i q}\right)$ in terms of width of the potential well. Indeed, noting that the quantity $\left(V_{v a p}-V_{l i q}\right)$ is an increasing function of the width of potential well, which itself increases when $n$ decreases, we remark that the value of the slope of the liquid-vapor coexistence curve decreases as $n$ is decreasing, for a given well depth $\varepsilon$.

For liquid metals, it should be noted that the repulsive part of the potential is always softer than for liquid rare gases. Moreover, even if $\varepsilon$ is slightly lower for metals than for rare gases, the report $\frac{\left(T_{C}-T_{T}\right) k_{B}}{\varepsilon}$ is much higher (between 2 and 4 ), which explains the elongation of the segment TC compared to that of rare gases. Also we mention that some flat-bottomed potentials[17, 18, 19] are likely to give a good description of the physical properties of elements having a low value of the ratio $\frac{T_{T}}{T_{C}}$. Such potentials are obviously not suitable for liquid rare gases, for which $\frac{T_{T}}{T_{C}} \simeq 0.56$, or organic and inorganic liquids, for which $0.25<\frac{T_{T}}{T_{C}}<0.45$. By contrast, they might be useful as empirical potentials for metals with low melting point, such as mercury, gallium, indium, tin, etc. with a ratio $\frac{T_{T}}{T_{C}}<0.1$.

\section{A Weak Electron-ion Interaction}

\subsection{The Schrödinger Equation}

The atoms of rare gases considered in the preceding subsection have electronic shells completely filled. This ensures the stability of their electronic structures and allows treating the rare gases as liquids composed of single atoms. In contrast, the atoms in liquid metals have few electrons in the outer envelope - the valence band or $s$ band. They are called the normal metals, such as alkali metals, which have a single electron in the $s$ band. The easiness with which they can lose the valence electron gives them a high electronic conductivity. The polyvalent metals with several valence electrons are also assumed to be normal metals. In contrast, the noble metals and transition metals that possess, in addition to the valence electrons, a narrow $d$ strip overlapping the $s$ band can not be treated like normal metals, because there are interactions ( $s$ - $d$ hybridization) between $s$ electrons and $d$ electrons participating in conduction.

In normal metals, the conduction electrons, separated from ions, constitute a free electron gas that plays an active role in determining the energy of the system while the core electrons, tightly bound to nuclei, have no major impact. These considerations put forward by Drude, a century ago, suggest to consider the liquid metal as a mixture of ions interacting within the free electron gas and to write the energy $E$ of the liquid metal as a sum of two terms:

$$
E=N u_{g}(V, T)+U_{N}\left(V, T, \mathbf{R}_{1}, \ldots, \mathbf{R}_{N}\right),
$$

where the first term is the energy contribution that depends on the temperature and volume of $N$ ions, and the second term is the energy contribution depending, in addition, on the configuration of ions in positions $\mathbf{R}_{i}$. It will be seen later that this contribution can be written as the sum of effective pair potentials:

$$
U_{N}\left(V, T, \mathbf{R}_{1}, \ldots, \mathbf{R}_{N}\right)=\frac{1}{2} \sum_{i} \sum_{j \neq i} u\left(R_{i j}\right),
$$

between entities (pseudoions), which differ noticeably from the genuine ions. The sum $\sum_{i} \sum_{j \neq i}$ and the factor $1 / 2$ come from the fact that the interaction between a pair of particles should not be counted twice. The effective potential $u\left(R_{i j}\right)$ has a functional form that depends on the volume and the temperature, as well as on the capacity of the electron gas to screen the ions. 
The calculation of the energy $E$ is a complicated problem of quantum mechanics solved by the perturbation theory to second order of the pseudopotential, i.e. the interaction between an electron and an ion.

Since a liquid metal is considered as a set of ions of charge $Z e$ (where $Z$ is the valence), imbedded in the free electron gas, its dynamic state can be described owing to the Schrdinger equation:

$$
H \psi=E \psi,
$$

where $H$ is the Hamiltonian of the system, $\psi$ the wave function and $E$ the energy. The Hamiltonian operator $H$ contains all the forms of energy such as kinetic energies of the free electrons and ions:

$$
\begin{aligned}
& T_{e l}=\sum_{i}\left(-\frac{\hbar^{2}}{2 m} \nabla_{i}^{2}\right), \\
& T_{i o}=\sum_{i}\left(-\frac{\hbar^{2}}{2 M} \nabla_{i}^{2}\right),
\end{aligned}
$$

and potential energies of the electron-electron, ion-ion and electron-ion interactions:

$$
\begin{aligned}
U_{e l-e l} & =\frac{1}{2} \sum_{i} \sum_{j \neq i} \frac{1}{4 \pi \varepsilon_{0}} \frac{e^{2}}{\left|\mathbf{r}_{i}-\mathbf{r}_{j}\right|} \\
& =\frac{1}{2} \sum_{i} \sum_{j \neq i} U_{e l}\left(\left|\mathbf{r}_{i}-\mathbf{r}_{j}\right|\right), \\
U_{i o-i o} & =\frac{1}{2} \sum_{\alpha} \sum_{\beta \neq \alpha} \frac{1}{4 \pi \varepsilon_{0}} \frac{Z^{2} e^{2}}{\left|\mathbf{R}_{\alpha}-\mathbf{R}_{\beta}\right|} \\
& =\frac{1}{2} \sum_{\alpha} \sum_{\beta \neq \alpha} U_{i o}\left(\left|\mathbf{R}_{\alpha}-\mathbf{R}_{\beta}\right|\right), \\
U_{e l-i o} & =-\sum_{i} \sum_{\alpha} \frac{1}{4 \pi \varepsilon_{0}} \frac{Z e^{2}}{\left|\mathbf{R}_{\alpha}-\mathbf{r}_{i}\right|} \\
& =\sum_{i} \sum_{\alpha} U_{e l-i o}\left(\left|\mathbf{R}_{\alpha}-\mathbf{r}_{i}\right|\right) .
\end{aligned}
$$

In such a scheme, the ions of mass $M$ have coordinates $\mathbf{R}_{\alpha}$ and the free electrons of charge $e$ and mass $m$ have coordinates $\mathbf{r}_{i}$.

To solve the Schrdinger equation (Eq. 49), it is essential to reduce the system of $(Z+1) N$ particles ${ }^{2}$ into interaction to a system of independent particles, with reasonable simplifications, this in order to write the wave function $\psi$ of the system as a product of wave functions of all the particles and the total energy $E$ as a sum of energies.

The first simplification (adiabatic approximation) takes into account the difference between the masses of the electrons and ions. The assumption made here is to admit that the ions are heavier and immobile with respect to the electrons whose

\footnotetext{
${ }^{2}$ Indeed, the metal is composed of $N$ ions and $N Z$ free electrons.

${ }^{3}$ The adiabatic approximation, known as the Born-Oppenheimer approximation, is usually presented in a more subtile manner, but the supposition that the ions are fixed affects very little the results of the energy
}

movements are much faster ${ }^{3}$. With this simplification, the ionion interaction energy $U_{i o-i o}$ must be calculated separately and added to the electronic energy $E_{e l}$ coming from the electronic Schrdinger equation:

$$
\begin{gathered}
\sum_{i}\left(-\frac{\hbar^{2}}{2 m} \nabla_{i}^{2}\right)+\frac{1}{2} \sum_{i} \sum_{j \neq i} U_{e l}\left(\left|\mathbf{r}_{i}-\mathbf{r}_{j}\right|\right) \\
+\sum_{i} \sum_{\alpha} U_{e l-i o}\left(\left|\mathbf{R}_{\alpha}-\mathbf{r}_{i}\right|\right) \psi_{e l}=E_{e l} \psi_{e l}
\end{gathered}
$$

where $\psi_{e l}$ represents the wave function of the electrons in presence of the ions.

A second simplification is needed to solve the above equation, which is not yet related to a single electron because of the double sum on $i$ and $j$ in the term $\sum_{i} \sum_{j \neq i} U_{e l}\left(\left|\mathbf{r}_{i}-\mathbf{r}_{j}\right|\right)$. Obviously, equation (55), describing the state of $Z N$ electrons interacting in the presence of the ions, could be reduced to an equation for one electron if there was no interaction between electrons $\left(U_{e l}=0\right)$ since the Hamiltonian would be decomposed into a sum of $Z N$ Hamiltonians. In the case of a system of interacting electrons, the reduction of equation (55) is however possible through the Hartree approximation. The latter consists in (1) approximating the many-electron wave function $\psi_{e l}$ as a product of single-particle functions:

$$
\psi_{e l}\left(\mathbf{r}_{1}, \mathbf{r}_{2}, \ldots, \mathbf{r}_{i}, \ldots\right)=\psi_{1}\left(\mathbf{r}_{1}\right) \psi_{2}\left(\mathbf{r}_{2}\right) \ldots \psi_{i}\left(\mathbf{r}_{i}\right) \ldots,
$$

and (2) replacing the energy of electron-electron interaction, $U_{e l-e l}$, by a function - the Hartree potential $W^{H}\left(r_{i}\right)$ representing the potential energy of the electron $i$ in the field of all the other electrons:

$$
\frac{1}{2} \sum_{i} \sum_{j \neq i} U_{e l}\left(\left|\mathbf{r}_{i}-\mathbf{r}_{j}\right|\right) \rightarrow \frac{1}{2} \sum_{i} W^{H}\left(r_{i}\right),
$$

where the Hartree potential satisfies the Poisson equation ${ }^{4}$ :

$$
\nabla^{2} W^{H}\left(r_{i}\right)=-\frac{e^{2}}{\varepsilon_{0}} \sum_{j \neq i}\left|\psi_{j}\left(r_{j}\right)\right|^{2} .
$$

The expression of $W^{H}\left(r_{i}\right)$ is:

$$
W^{H}\left(r_{i}\right)=\sum_{j \neq i} \int \frac{e^{2}}{4 \pi \varepsilon_{0}} \frac{\left|\psi_{j}\left(r_{j}\right)\right|^{2}}{\left|\mathbf{r}_{i}-\mathbf{r}_{j}\right|} d \mathbf{r}_{j},
$$

where its physical meaning is as follows: (1) $e\left|\psi_{j}\left(r_{j}\right)\right|^{2}$ represents the charge density of the electron cloud produced by the electron $j$, (2) the quantity $e\left|\psi_{j}\left(r_{j}\right)\right|^{2} d \mathbf{r}_{j}$ is the electric charge of the volume element $d \mathbf{r}_{j}$ and (3) the integral $\int \frac{e^{2}}{4 \pi \varepsilon_{0}} \frac{\left|\psi_{j}\left(r_{j}\right)\right|^{2}}{\left|\mathbf{r}_{i}-\mathbf{r}_{j}\right|} d \mathbf{r}_{j}$ is the interaction energy of the electron $i$

calculation.

${ }^{4}$ The Poisson and Coulomb equations are written in the rationalized M.K.S. unit system (SI units) as $\nabla^{2} \Phi(\mathbf{r}) \propto-\frac{1}{\varepsilon_{0}}$ and $F(\mathbf{r}) \propto \frac{1}{4 \pi \varepsilon_{0}}$. 
with the electron $j$. Note that the potential energy $W^{H}\left(r_{i}\right)$ of the electron $i$ depends not only on the movements of all the other electrons but, indirectly, on its own motion, which consequently influences the movements of the other electrons. In view of this, the function $W^{H}\left(r_{i}\right)$ determines not only the motion of the electron $i$ but also depends on it. The determination of the function $W^{H}\left(r_{i}\right)$ is made, in principle, in a self-consistent manner by iterative calculations.

Thus, the implementation of the Hartree approximation enables us to write the Hamiltonian of equation (55) as:

$$
H_{e l}=\sum_{i}\left[-\frac{\hbar^{2}}{2 m} \nabla_{i}^{2}+W^{H}\left(r_{i}\right)+\sum_{\alpha} U_{e l-i o}\left(\left|\mathbf{R}_{\alpha}-\mathbf{r}_{i}\right|\right)\right],
$$

and to reduce the Schrdinger equation for multiple electrons (Eq. 55) to the equation for a single electron:

$$
\left[-\frac{\hbar^{2}}{2 m} \nabla_{i}^{2}+W^{H}\left(r_{i}\right)+\sum_{\alpha} U_{e l-i o}\left(\left|\mathbf{R}_{\alpha}-\mathbf{r}_{i}\right|\right)\right] \psi_{i}=E_{i} \psi_{i}
$$

where the wave function of the system $\psi_{e l}$ is equal to the product of the single wave functions $\left(\psi_{e l}=\prod_{i} \psi_{i}\right)$ and the electronic energy of the system is equal to the sum of the energies of each electron $\left(E_{e l}=\sum_{i} E_{i}\right)$.

It should be mentioned that the Fermi statistics can be incorporated into the Hartree approximation in replacing the product of single wave functions by orbitals under the form of a Slater determinant. This new scheme, known as the Hartree-Fock approximation, leads to an additional term in the Schr dinger equation taking account of the exchange effects, which improves the total energy computation. Note that the correlation effects can also be included in the calculations. In the following, we will introduce the exchange and correlation effects through the screening and pseudopotential theories, without resorting to the Hartree-Fock approximation.

\subsection{Concept of Pseudopotential}

The two foregoing simplifications have been made to reduce the study of liquid metals to that of an independent electron. But the resolution of equation (61) is not yet possible because the electron-ion interaction energy $U_{e l-i o}(r)$ and the Hartree potential $W^{H}(r)$ are unknown functions.

Consider first the potential of electron-ion interaction. If the electron $i$ is close to the ion $\alpha$, the most important energy contribution is provided by the electrostatic potential between the electron $i$ and the ion $\alpha$ (Figure 5). This potential has a deep well surrounding the ion and varies as $1 / r$ when the electron moves away. Regarding the interactions between the electron $i$ and all the other ions, they are very weak when the distance separating them is large. Thus they provide no significant contribution to the energy $E_{i}$, so that the dominant feature of the sum $\sum_{\alpha} U_{e l-i o}\left(\left|\mathbf{R}_{\alpha}-\mathbf{r}_{i}\right|\right)$ is to depend only on the interaction between the electron $i$ and the nearest ion.

To solve the Schrdinger equation for one electron (Eq. 61 ), the perturbation theory independent of time is required. This method can be implemented only when the potential energy operator is small enough to be treated as a perturbation. However, this is not the case because of the divergence of the potential $\sum_{\alpha} U_{e l-i o}\left(\left|\mathbf{R}_{\alpha}-\mathbf{r}_{i}\right|\right)$ for $\mathbf{r}_{i} \simeq \mathbf{R}_{\alpha}$. To bypass this difficulty, the true wave function $\psi_{i}$ is replaced by a pseudofunction $\chi_{i}$, smooth on the outside and the inside of the ion, with the aim of transforming the potential energy operator, $\sum_{\alpha} U_{e l-i o}\left(\left|\mathbf{R}_{\alpha}-\mathbf{r}_{i}\right|\right)$, into a pseudopotential $W_{0}(r)=$ $\sum_{\alpha}^{\alpha} w_{0}\left(\left|\mathbf{R}_{\alpha}-\mathbf{r}_{i}\right|\right)$ sufficiently small everywhere to justify the use of the perturbation theory.

The pseudopotential theory proposed by Phillips and Kleinman[1] is based on theoretical foundations that will not be discussed here. In turn, we provide a justification to the fact that the pseudopotential $W_{0}(r)$ is small throughout the system, and give a simple analytical expression for the individual pseudopotential $w_{0}(r)$ based on the following arguments. As already mentioned, the potential felt by the electron $i$ in the vicinity of the ion $\alpha$ is a Coulomb potential equal to $-\frac{1}{4 \pi \varepsilon_{0}} \frac{Z e^{2}}{\left|\mathbf{R}_{\alpha}-\mathbf{r}_{i}\right|}$. But the electron $i$ is also subjected to a repulsive potential generated by the core electrons of the ion $\alpha$ due to the Pauli exclusion principle, which can be represented by a Dirac peak $+\lambda \delta\left(\left|\mathbf{R}_{\alpha}-\mathbf{r}_{i}\right|\right.$ given the small size of ions. Therefore, in the vicinity of an ion, the pseudopotential $w_{0}(r)$ may be taken to be the sum of these two contributions (Figure 5) using, for instance, the representation of the analytical model of Ashcroft[20]:

$$
w_{0}(r)= \begin{cases}0 & \text { if } r<R_{c} \\ -\frac{1}{4 \pi \varepsilon_{0}} \frac{Z e^{2}}{r} & \text { if } r>R_{c}\end{cases}
$$

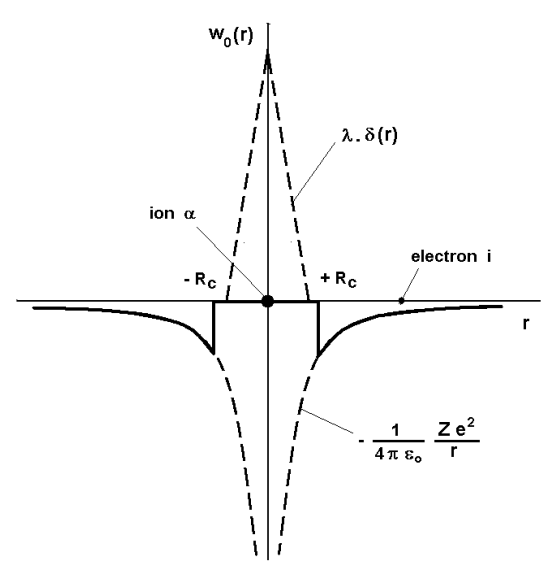

Figure 5. Analytical model of the Ashcroft pseudopotential. 
In this model, it is assumed that the repulsive potential cancels completely the attractive potential in a sphere of radius $R_{c}$ to be determined. Obviously, there are models in which the potential in the core is not zero. Some, like that of Ashcroft, has a simple analytical form. Others are operators (non-local pseudopotentials) and, therefore, have no simple analytical form [21-24]. All calculations presented in the sequel will be made with the Ashcroft local pseudopotential.

If it is accepted that a low pseudopotential $W_{0}(r)$ exists, the problem of one free electron in the collection of the ions and other electrons might be then solved. To do this, we rewrite equation (61) with the pseudopotential $W_{0}(r)$ and the pseudo wave function $\chi_{k}(r)$, in removing the index $i$ and using the classical notations of quantum mechanics ${ }^{5}$ :

$$
\left[-\frac{\hbar^{2}}{2 m} \nabla^{2}+W(r)\right] \chi_{k}(r)=E(k) \chi_{k}(r),
$$

with:

$$
W(r)=W^{H}(r)+W_{0}(r) .
$$

Then, the electronic energy $E(k)$ may be determined with the perturbation theory by adopting the free electron model as zero order approximation. This model states that the Schrdinger equation of one free electron in a box of volume $V$ is written:

$$
-\frac{\hbar^{2}}{2 m} \nabla^{2} \chi_{k}^{0}(r)=E^{0}(k) \chi_{k}^{0}(r)
$$

It is found that this equation admits a solution under the form of a plane wave :

$$
\chi_{k}^{0}(\mathbf{r})=\frac{1}{\sqrt{V}} \exp (i \mathbf{k} \cdot \mathbf{r})
$$

and has an energy spectrum given by:

$$
E^{0}(k)=\frac{\hbar^{2} k^{2}}{2 m} .
$$

Thus, in the zero order approximation, the energy of one free electron $E^{0}(k)$ varies continuously with the square of the wave vector $\mathbf{k}$. Moreover, the electronic energy of the system composed of $N Z$ free electrons is:

$$
E=\frac{3}{5} N Z \frac{\hbar^{2} k_{F}^{2}}{2 m}
$$

where $k_{F}=\left(\frac{3 \pi^{2} Z N}{V}\right)^{1 / 3}$ is the wave vector at the Fermi level.

\footnotetext{
${ }^{5}$ The vector $\mathbf{k}$ characterizes any given wave function and must appear as index within this function.

${ }^{6}$ The reader may consults any book of quantum mechanics or articles in open access at the addresses: http://www.freebookcentre.net/Physics/Quantum-Mechanics-Books.html and specially:

http://www.lsr.ph.ic.ac.uk/ plenio/lecture.pdf
}

\section{Form Factor, Structure Factor and Electron Energy}

\subsection{Perturbation Theory for non Degenerate States}

The perturbation theory aims to determine the corrections to the energy $E^{0}(k)$ and wave function $\chi_{k}^{0}(\mathbf{r})$ of the free electron system, when a perturbation $W(r)$ (Eq. 64) is imposed. In this section, we briefly describe the time independent perturbation theory to second order ${ }^{6}$ for non degenerate states, because it is the only useful result here.

Consider the unperturbed quantum system governed by the Schrdinger equation:

$$
\hat{H} \varphi_{\alpha}^{0}(\mathbf{r})=E_{\alpha}^{0} \varphi_{\alpha}^{0}(\mathbf{r}),
$$

where the discrete spectrum of eigenvalues $E_{\alpha}^{0}$ and eigenfunctions $\varphi_{\alpha}^{0}(\mathbf{r})$ of the Hamiltonian $\hat{H}$ are assumed to be known. As the quantum states are not degenerate, only one wave function corresponds to each value of energy. In what follows, it is proposed to determine the approximate solutions of the wave function $\psi_{\beta}(\mathbf{r})$ and energy $E_{\beta}$ of the quantum system subjected to the weak perturbation $\hat{W}$, whose equation Schrdinger is:

$$
(\hat{H}+\lambda \hat{W}) \psi_{\beta}(\mathbf{r})=E_{\beta} \psi_{\beta}(\mathbf{r})
$$

where $\lambda$ is a parameter primarily used to facilitate the analysis. It takes the value 0 when the system is not perturbed and 1 when the system is perturbed.

To find the solution of this equation, use is made of the Dirac prescription that consists in expanding the unknown wave function $\psi_{\beta}(\mathbf{r})$ on the basis of the eigenwave functions $\varphi_{\alpha}^{0}(\mathbf{r})$, namely:

$$
\psi_{\beta}(\mathbf{r})=\sum_{\alpha} c_{\beta \alpha} \varphi_{\alpha}^{0}(\mathbf{r})
$$

This expansion is then substituted in equation (70):

$$
\sum_{\alpha} c_{\beta \alpha}(\hat{H}+\lambda \hat{W}) \varphi_{\alpha}^{0}(\mathbf{r})=\sum_{\alpha} c_{\beta \alpha} E_{\beta} \varphi_{\alpha}^{0}(\mathbf{r})
$$

which, with equation (69), may be put in the form:

$$
\sum_{\alpha} c_{\beta \alpha}\left(E_{\alpha}^{0}+\lambda \hat{W}\right) \varphi_{\alpha}^{0}(\mathbf{r})=\sum_{\alpha} c_{\beta \alpha} E_{\beta} \varphi_{\alpha}^{0}(\mathbf{r}) .
$$

Multiply now the left hand side by $\varphi_{\gamma}^{0 *}$ and integrate over the domain concerned. The result is: 


$$
\sum_{\alpha} c_{\beta \alpha} \int \varphi_{\gamma}^{0 *} E_{\alpha}^{0} \varphi_{\alpha}^{0} d^{3} r+\sum_{\alpha} c_{\beta \alpha} \int \varphi_{\gamma}^{0 *} \lambda \hat{W} \varphi_{\alpha}^{0} d^{3} r=\sum_{\alpha} c_{\beta \alpha} \int \varphi_{\gamma}^{0 *} E_{\beta} \varphi_{\alpha}^{0} d^{3} r
$$

And after simplifications ${ }^{7}$, this relation becomes:

$$
\sum_{\alpha} c_{\beta \alpha} \lambda W_{\gamma \alpha}=\left(E_{\beta}-E_{\gamma}^{0}\right) c_{\beta \gamma}
$$

The perturbation theory sets itself as a goal to seek the energy $E_{\beta}$ and the coefficients $c_{\beta \alpha}$, likely to solve equation (70), under the series expansions:

$$
\begin{aligned}
E_{\beta} & =E_{\beta}^{0}+\lambda E_{\beta}^{(1)}+\lambda^{2} E_{\beta}^{(2)}+\ldots, \\
c_{\beta \alpha} & =c_{\beta \alpha}^{0}+\lambda c_{\beta \alpha}^{(1)}+\lambda^{2} c_{\beta \alpha}^{(2)}+\ldots,
\end{aligned}
$$

where the quantities $E_{\beta}^{0}$ and $c_{\beta \alpha}^{0}$ correspond to the unperturbed system (zero order approximation), $E_{\beta}^{(1)}$ and $c_{\beta \alpha}^{(1)}$ to the first order corrections, etc. By inserting the previous expansions in equation (75), it comes:

$$
\sum_{\alpha}\left[c_{\beta \alpha}^{0}+\lambda c_{\beta \alpha}^{(1)}+\ldots\right] \lambda W_{\gamma \alpha}=\left(E_{\beta}^{0}+\lambda E_{\beta}^{(1)}+\lambda^{2} E_{\beta}^{(2)}+\ldots-E_{\gamma}^{0}\right)\left[c_{\beta \gamma}^{0}+\lambda c_{\beta \gamma}^{(1)}+\lambda^{2} c_{\beta \gamma}^{(2)}+\ldots\right] .
$$

When the system is not perturbed $(\lambda=0)$, this relation reduces to $\left(E_{\beta}^{0}-E_{\gamma}^{0}\right) c_{\beta \gamma}^{0}=0$ implying that $c_{\beta \gamma}^{0}=0$ when $\beta \neq \gamma$. In addition, since equation (71) reads:

$$
\psi_{\beta}(\mathbf{r})=\sum_{\alpha} c_{\beta \alpha}^{0} \varphi_{\alpha}^{0}(\mathbf{r})=\varphi_{\beta}^{0}(\mathbf{r})
$$

it follows that the coefficient $c_{\beta \alpha}^{0}$ identifies with the Kronecker delta symbol, i.e. $c_{\beta \alpha}^{0}=\delta_{\beta \alpha}$, that is worth 0 when $\alpha \neq \beta$ and 1 when $\alpha=\beta$.

To determine the first order correction of the perturbed system, we neglect the powers of $\lambda$ greater than or equal to

two in equation (78) and take $\lambda=1$ :

$$
\sum_{\alpha} \delta_{\beta \alpha} W_{\gamma \alpha}=\left(E_{\beta}^{0}-E_{\gamma}^{0}\right)\left[\delta_{\beta \gamma}+c_{\beta \gamma}^{(1)}\right]+E_{\beta}^{(1)} \delta_{\beta \gamma}
$$

It results from the above relation that:

$$
\begin{aligned}
& \text { if } \gamma=\beta \text { and } \alpha \neq \beta, \quad E_{\beta}^{(1)}=0, \\
& \text { if } \gamma=\beta \text { and } \alpha=\beta, \quad E_{\beta}^{(1)}=W_{\beta \beta}=\int \varphi_{\beta}^{0 *} \hat{W} \varphi_{\beta}^{0} d^{3} r, \\
& \text { if } \gamma \neq \beta \text { and } \alpha=\beta, \quad W_{\gamma \beta}=\left(E_{\beta}^{0}-E_{\gamma}^{0}\right) c_{\beta \gamma}^{(1)} .
\end{aligned}
$$

With these conditions in mind, the first order approximation of the energy $E_{\beta}$ (Eq. 76) and wave function $\psi_{\beta}(\mathbf{r})($ Eq. 71) of the perturbed system are written:

$$
\begin{aligned}
E_{\beta} & =E_{\beta}^{0}+W_{\beta \beta} \\
\psi_{\beta}(\mathbf{r}) & =\sum_{\alpha}\left[\delta_{\beta \alpha}+c_{\beta \alpha}^{(1)}\right] \varphi_{\alpha}^{0}(\mathbf{r})=\varphi_{\beta}^{0}(\mathbf{r})+\sum_{\alpha \neq \beta} \frac{W_{\alpha \beta}}{E_{\beta}^{0}-E_{\alpha}^{0}} \varphi_{\alpha}^{0}(\mathbf{r}) .
\end{aligned}
$$

Then, to determine the second order correction of the perturbed system, we ignore the powers of $\lambda$ greater than or equal to three in equation ( 78) and take $\lambda=1$ :

$$
\sum_{\alpha}\left[\delta_{\beta \alpha}+c_{\beta \alpha}^{(1)}\right] W_{\gamma \alpha}=\left(E_{\beta}^{0}-E_{\gamma}^{0}\right)\left[\delta_{\beta \gamma}+c_{\beta \gamma}^{(1)}+c_{\beta \gamma}^{(2)}\right]+E_{\beta}^{(1)}\left[\delta_{\beta \gamma}+c_{\beta \gamma}^{(1)}\right]+E_{\beta}^{(2)} \delta_{\beta \gamma} .
$$

\footnotetext{
${ }^{7}$ Since the base formed by the eigenfunctions $\psi_{\alpha}^{0}$ is orthonormal, the three terms of the previous equation are reduced, respectively, to:

$$
\begin{aligned}
\sum_{\alpha} c_{\beta \alpha} \int \varphi_{\gamma}^{0 *} E_{\alpha}^{0} \varphi_{\alpha}^{0} d^{3} r & =c_{\beta \gamma} E_{\gamma}^{0}, \\
\sum_{\alpha} c_{\beta \alpha} \int \varphi_{\gamma}^{0 *} E_{\beta} \varphi_{\alpha}^{0} d^{3} r & =c_{\beta \gamma} E_{\beta}, \\
\sum_{\alpha} c_{\beta \alpha} \int \varphi_{\gamma}^{0 *} \hat{W} \varphi_{\alpha}^{0} d^{3} r & =\sum_{\alpha} c_{\beta \alpha} W_{\gamma \alpha},
\end{aligned}
$$
}


In the case where $\gamma=\beta$ with $\beta \neq \alpha$, for which $E_{\beta}^{(1)}=0$, the above relation reduces to:

$$
E_{\beta}^{(2)}=\sum_{\alpha \neq \beta} c_{\beta \alpha}^{(1)} W_{\beta \alpha}
$$

Now, in substituting $c_{\beta \alpha}^{(1)}$ in the previous equation, with the help of equation (82), we obtain the energy to second order:

$$
E_{\beta}=E_{\beta}^{0}+W_{\beta \beta}+\sum_{\alpha \neq \beta} \frac{W_{\alpha \beta} W_{\beta \alpha}}{E_{\beta}^{0}-E_{\alpha}^{0}} .
$$

\subsection{Factorization of the Matrix Elements}

In the study of liquid metals, it is not necessary to go beyond the approximations to second order for the energy (Eq. 87) and first order for the wave function (Eq. 84). Consequently, the energy $E(k)$ and the wave function $\chi_{k}(r)$ of one conduction electron, in the system subjected to the perturbation $W(r)$, can be evaluated in rewriting equations (87) and (84) with the notations for the energy and the matrix element of the perturbation adapted to the problem:

$$
E(k)=\frac{\hbar^{2} k^{2}}{2 m}+\langle\mathbf{k}|W| \mathbf{k}\rangle+\sum_{q \neq 0} \frac{|\langle\mathbf{k}+\mathbf{q}|W| \mathbf{k}\rangle|^{2}}{\frac{\hbar^{2}}{2 m}\left[\mathbf{k}^{2}-|\mathbf{k}+\mathbf{q}|^{2}\right]},
$$

$$
\begin{aligned}
\chi_{k}(r) & =\frac{1}{\sqrt{V}} \exp (i \mathbf{k} \cdot \mathbf{r})+\sum_{q \neq 0} \frac{|\langle\mathbf{k}+\mathbf{q}|W| \mathbf{k}\rangle|}{\frac{\hbar^{2}}{2 m}\left[\mathbf{k}^{2}-|\mathbf{k}+\mathbf{q}|^{2}\right]} \\
& \times \frac{1}{\sqrt{V}} \exp [i(\mathbf{k}+\mathbf{q}) \cdot \mathbf{r}]
\end{aligned}
$$

where the first terms of these series correspond to the energy and the wave function of the free electron model (Eqs. 66 and 67), while the corrective terms depend on the matrix element:

$$
\begin{aligned}
\langle\mathbf{k}+\mathbf{q}|W| \mathbf{k}\rangle & =\frac{1}{V} \int \exp [-i(\mathbf{k}+\mathbf{q}) \cdot \mathbf{r}] \\
& \times W(r) \exp (i \mathbf{k} \cdot \mathbf{r}) d \mathbf{r}
\end{aligned}
$$

which is responsible for the diffusion over a surface of constant energy. Indeed, $\langle\mathbf{k}+\mathbf{q}|W| \mathbf{k}\rangle$ is the expression of the diffusion of a conduction electron from the initial state defined by the wave vector $\mathbf{k}$ to the final state $\mathbf{k}^{\prime}=\mathbf{k}+\mathbf{q}$, by the total pseudopotential $W(r)$ weakly scattering. Since the matrix element must be calculated with the pseudopotential $W_{0}(r)$ of the bare ion plus the Hartree potential ${ }^{8} W^{H}(r)$ (Eq. 64), the total potential $W(r)$ is written as a sum of individual pseudopotentials centered on the ions, under the form :

$$
W(r)=W^{H}(r)+W_{0}(r)=\sum_{\alpha} w\left(\left|\mathbf{R}_{\alpha}-\mathbf{r}\right|\right) .
$$

Finally, the expression of the matrix element is found in inserting equation (91) in equation (90):

$$
\begin{aligned}
\langle\mathbf{k}+\mathbf{q}|W| \mathbf{k}\rangle & =\frac{1}{V} \int \exp [-i(\mathbf{k}+\mathbf{q}) \cdot \mathbf{r}] \times \sum_{\alpha} w\left(\left|\mathbf{R}_{\alpha}-\mathbf{r}\right|\right) \exp (i \mathbf{k} \cdot \mathbf{r}) d \mathbf{r} \\
& =\frac{1}{V} \sum_{\alpha} \exp \left(-i \mathbf{q} \cdot \mathbf{R}_{\alpha}\right) \int \exp \left[-i(\mathbf{k}+\mathbf{q}) \cdot\left(\mathbf{r}-\mathbf{R}_{\alpha}\right)\right] w\left(\left|\mathbf{R}_{\alpha}-\mathbf{r}\right|\right) \times \exp \left[i \mathbf{k} \cdot\left(\mathbf{r}-\mathbf{R}_{\alpha}\right)\right] d \mathbf{r}
\end{aligned}
$$

Since $\left(\mathbf{R}_{\alpha}-\mathbf{r}\right)$ is a dummy variable, we pose $\mathbf{X}=\mathbf{r}-\mathbf{R}_{\alpha}$ in order to achieve the factorization of the matrix element as:

$$
\langle\mathbf{k}+\mathbf{q}|W| \mathbf{k}\rangle=\frac{1}{N} \sum_{\alpha} \exp \left(-i \mathbf{q} \cdot \mathbf{R}_{\alpha}\right)\langle\mathbf{k}+\mathbf{q}|w| \mathbf{k}\rangle,
$$

with:

$$
\langle\mathbf{k}+\mathbf{q}|w| \mathbf{k}\rangle=\frac{N}{V} \int \exp [-i(\mathbf{k}+\mathbf{q}) \cdot \mathbf{X}] \times w(|\mathbf{X}|) \exp (i \mathbf{k} \cdot \mathbf{X}) d \mathbf{X} .
$$

It should be stressed that the square of the matrix element in equation (88) is usually written as:

$$
\langle\mathbf{k}+\mathbf{q}|W| \mathbf{k}\rangle^{2}=\frac{1}{N^{2}} \sum_{\alpha, \beta} \exp \left[-i \mathbf{q} \cdot\left(\mathbf{R}_{\beta}-\mathbf{R}_{\alpha}\right)\right]\langle\mathbf{k}+\mathbf{q}|w| \mathbf{k}\rangle^{2}=\frac{1}{N} S(q)\langle\mathbf{k}+\mathbf{q}|w| \mathbf{k}\rangle^{2}
$$

where $\langle\mathbf{k}+\mathbf{q}|w| \mathbf{k}\rangle$ is the form factor resulting from the individual potential of the ion and $S(q)$ the ionic structure factor, which depends on the position of the ions and can be directly measured by X-ray or neutron scattering. Its definition is:

$$
S(q)=\frac{1}{N} \sum_{\alpha, \beta} \exp \left[-i \mathbf{q} \cdot\left(\mathbf{R}_{\beta}-\mathbf{R}_{\alpha}\right)\right]
$$

\footnotetext{
${ }^{8}$ The Hartree potential is subordinate to the rearrangement of the electron gas around the ions forming an electronic screen.
} 
In the framework of the perturbation theory briefly presented, it is found that the electron energy $E_{e l}$ of a metal is equal to the sum of the energies $E(k)$ of all the free electrons. In practice, the perturbation theory performs the calculations in the reciprocal space and not in the direct space. Therefore, the electronic energy $E_{e l}$ is calculated in k-space in doing the sum of all the energy states occupied in the Fermi sphere of radius $k_{F}$, with the expression ${ }^{9}$ :

$$
E_{e l}=\sum_{k \leq k_{F}} E(k)=\frac{2 V}{(2 \pi)^{3}} \int_{k \leq k_{F}} E(k) d^{3} k,
$$

where $E(k)$ is given by equation (88). The electron energy $E_{e l}$ of a metal may thus be put under the form ${ }^{10}$ :

$$
E_{e l}=\sum_{k \leq k_{F}} E(k)=\frac{2 V}{(2 \pi)^{3}} \int_{k \leq k_{F}}\left[\frac{\hbar^{2} k^{2}}{2 m}+\langle\mathbf{k}|W| \mathbf{k}\rangle+\sum_{q \neq 0} \frac{|\langle\mathbf{k}+\mathbf{q}|W| \mathbf{k}\rangle|^{2}}{\frac{\hbar^{2}}{2 m}\left[\mathbf{k}^{2}-|\mathbf{k}+\mathbf{q}|^{2}\right]}\right] d^{3} k .
$$

\section{Screening Theory in the Hartree Approximation}

\subsection{Form Factor of the Ashcroft Pseudopotential}

As a rough guide, the form factor of Ashcroft's pseudopotential $w_{0}(r)$ will be calculated by substituting equation (62) in equation (94):

$$
\left\langle\mathbf{k}+\mathbf{q}\left|w_{0}\right| \mathbf{k}\right\rangle=\frac{N}{V} \iiint \exp [-i(\mathbf{k}+\mathbf{q}) \cdot \mathbf{r}] \times w_{0}(r) \exp (i \mathbf{k} \cdot \mathbf{r}) d^{3} r
$$

The integration in the above relation is better carried out with the spherical coordinates:

$$
\begin{aligned}
d^{3} r & =r^{2} \sin \theta d \theta d \varphi d r, \\
\text { and } \quad \mathbf{q r} & =q r \cos \theta,
\end{aligned}
$$

after placing the vectors $\mathbf{q}$ and $\mathbf{r}$ as shown in figure 6.

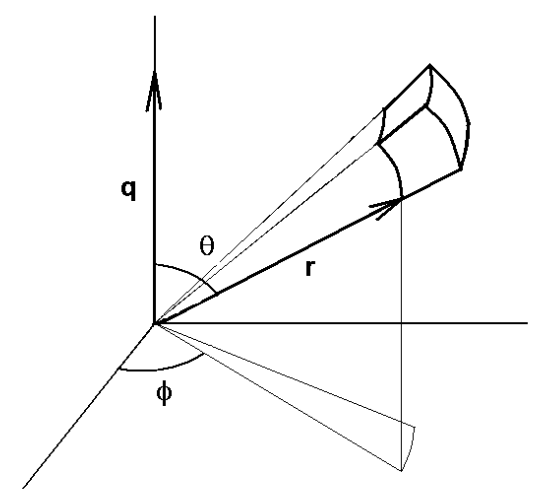

Figure 6. Positions of the vectors $\mathbf{q}$ and $\mathbf{r}$ to calculate the form factor $w_{0}(q)$.

${ }^{9}$ The transformation of the discrete sum into an integral over $k$ is done by using the formula:

$$
\sum_{k \leq k_{F}} \rightarrow \frac{2 V}{(2 \pi)^{3}} \int_{k \leq k_{F}} d^{3} k
$$

The number of electrons in the unit volume of the $\mathbf{k}$-space is $\frac{2 V}{(2 \pi)^{3}}$. However, for a free electron system, the volume occupied by the electrons in $\mathbf{k}$-space is a sphere of radius $k_{F}$, called the Fermi sphere. Therefore, the number of free electrons $N Z$ is the product of the number of electrons per unit volume multiplied by the volume of the Fermi sphere:

$$
N Z=\frac{2 V}{(2 \pi)^{3}} \int_{k \leq k_{F}} d^{3} k=\frac{2 V}{(2 \pi)^{3}} \frac{4}{3} \pi k_{F}^{3},
$$

hence

$$
k_{F}=\left(\frac{3 \pi^{2} N Z}{V}\right)^{1 / 3} .
$$

${ }^{10}$ From now, it is important to note that the energy $E_{e l}$ does not correspond exactly to the electronic energy of the metal because, to perform its calculation, the electron-electron energy is counted twice. Therefore, the latter should be subtracted at any time. 
With this change of variables, the form factor becomes:

$$
\left\langle\mathbf{k}+\mathbf{q}\left|w_{0}\right| \mathbf{k}\right\rangle=2 \pi \frac{N}{V} \iint \exp (-i q r \cos \theta) \times w_{0}(r) r^{2} \sin \theta d \theta d r .
$$

To perform the integration on the variable $\theta$, we take $\mu=\cos \theta$ ranging from 1 to -1 , with $d \mu=-\sin \theta d \theta$, which gives:

$$
\left\langle\mathbf{k}+\mathbf{q}\left|w_{0}\right| \mathbf{k}\right\rangle=2 \pi \frac{N}{V} \int\left[-\int_{1}^{-1} \exp (-i q r \mu) d \mu\right] \times w_{0}(r) r^{2} d r
$$

where the integral in square brackets is:

$$
\left[-\int_{1}^{-1} \exp (-i q r \mu) d \mu\right]=-\frac{1}{i q r}[\exp (-i q r)-\exp (i q r)]
$$

Before performing the integration over $r$, we replace the pseudopotential by its expression (Eq. 62) and introduce the damping factor $\exp (-\alpha r)$ that will disappear at the end of the calculation by letting $\alpha$ go toward zero, as follows:

$$
\begin{aligned}
\left\langle\mathbf{k}+\mathbf{q}\left|w_{0}\right| \mathbf{k}\right\rangle & =-\frac{2 \pi}{i q} \frac{N}{V} \\
& \times\left\{\int_{R_{c}}^{\infty} \frac{1}{r} \exp [-(\alpha+i q) r]\left(-\frac{1}{4 \pi \varepsilon_{0}} \frac{Z e^{2}}{r}\right) r^{2} d r-\int_{R_{c}}^{\infty} \frac{1}{r} \exp [-(\alpha-i q) r]\left(-\frac{1}{4 \pi \varepsilon_{0}} \frac{Z e^{2}}{r}\right) r^{2} d r\right\} \\
& =\frac{2 \pi}{i q} \frac{N}{V} \frac{Z e^{2}}{4 \pi \varepsilon_{0}}\left\{\int_{R_{c}}^{\infty} \exp [-(\alpha+i q) r] d r-\int_{R_{c}}^{\infty} \exp [-(\alpha-i q) r] d r\right\}
\end{aligned}
$$

The next integration over $r$, with $\alpha=0$, gives:

$$
\begin{aligned}
\left\langle\mathbf{k}+\mathbf{q}\left|w_{0}\right| \mathbf{k}\right\rangle & =\frac{2 \pi}{i q} \frac{N}{V} \frac{Z e^{2}}{4 \pi \varepsilon_{0}} \times\left\{\frac{1}{(\alpha+i q)} \exp \left[-(\alpha+i q) R_{c}\right]-\frac{1}{(\alpha-i q)} \exp \left[-(\alpha-i q) R_{c}\right]\right\} \\
& =\frac{2 \pi}{(i q)^{2}} \frac{N}{V} \frac{Z e^{2}}{4 \pi \varepsilon_{0}}\left\{\exp \left(-i q R_{c}\right)+\exp \left(i q R_{c}\right)\right\} \\
& =-\frac{Z e^{2}}{\varepsilon_{0} q^{2}} \frac{N}{V} \cos \left(q R_{c}\right)
\end{aligned}
$$

\subsection{Matrix Element of the Hartree Potential}

In the preceding subsections, the expression of the electronic energy $E_{e l}=\sum_{k<k_{F}} E(k)$ of metals has been derived as a function of $E(k)$ (Eq. 98). The energy $E(k)$ is the energy of one given conduction electron in interaction with the ions and the other electrons of the system, and it depends on the total pseudopotential $W(\mathbf{r})=W^{H}(\mathbf{r})+W_{0}(\mathbf{r})$ via the matrix element $\langle\mathbf{k}+\mathbf{q}|W| \mathbf{k}\rangle$ (Eq. 90). However, this matrix element is only partially known. Indeed, there is an expression for the matrix element $\left\langle\mathbf{k}+\mathbf{q}\left|W_{0}\right| \mathbf{k}\right\rangle$ of the bare pseudopotential (Eq. 106), but not any for the matrix element $\left\langle\mathbf{k}+\mathbf{q}\left|W^{H}\right| \mathbf{k}\right\rangle$ containing the electron-electron interactions in the Hartree approximation.

To find the expression of the matrix element $\left\langle\mathbf{k}+\mathbf{q}\left|W^{H}\right| \mathbf{k}\right\rangle$, use is made of the screening theory[25] based on the concept of dielectric function. The principle of the method is to determine, in a self-consistent manner, the matrix element $\left\langle\mathbf{k}+\mathbf{q}\left|W^{H}\right| \mathbf{k}\right\rangle$ and the matrix element $\langle\mathbf{k}+\mathbf{q}|W| \mathbf{k}\rangle=\left\langle\mathbf{k}+\mathbf{q}\left|W^{H}\right| \mathbf{k}\right\rangle+\left\langle\mathbf{k}+\mathbf{q}\left|W_{0}\right| \mathbf{k}\right\rangle$ as a function of the electronic cloud density $n(\mathbf{r})$. The method consists of calculating the electron density with the wave function (first postulate of quantum mechanics) by jointly using the Poisson equation. Thus, the electron density $n(\mathbf{r})$ is given by the sum, over all occupied states in the Fermi sphere, of the product of the pseudo wave functions $\chi_{k}(\mathbf{r})$ (Eq. 89) by its complex conjugate $\chi_{k}^{*}(\mathbf{r})$, that is to say:

$$
n(\mathbf{r})=\sum_{k \leq k_{F}} \chi_{k}(\mathbf{r}) \chi_{k}^{*}(\mathbf{r}),
$$

or:

$$
n(\mathbf{r})=\sum_{k \leq k_{F}}\left[\frac{1}{\sqrt{V}} \exp (i \mathbf{k} \cdot \mathbf{r})+\sum_{q \neq 0} \frac{a_{q}(\mathbf{k})}{\sqrt{V}} \exp [i(\mathbf{k}+\mathbf{q}) \cdot \mathbf{r}]\right] \times\left[\frac{1}{\sqrt{V}} \exp (-i \mathbf{k} \cdot \mathbf{r})+\sum_{q \neq 0} \frac{a_{q}^{*}(\mathbf{k})}{\sqrt{V}} \exp [-i(\mathbf{k}+\mathbf{q}) \cdot \mathbf{r}]\right]
$$

where it has been written:

$$
a_{q}(\mathbf{k})=\frac{|\langle\mathbf{k}+\mathbf{q}|W| \mathbf{k}\rangle|}{\frac{\hbar^{2}}{2 m}\left[\mathbf{k}^{2}-|\mathbf{k}+\mathbf{q}|^{2}\right]}
$$


so that the total electron density is:

$$
n(\mathbf{r})=\frac{1}{V} \sum_{k \leq k_{F}}\left[1+\sum_{q \neq 0} a_{q}(\mathbf{k}) \exp (i \mathbf{q} \cdot \mathbf{r})+\sum_{q \neq 0} a_{q}^{*}(\mathbf{k}) \exp (-i \mathbf{q} \cdot \mathbf{r})+\ldots\right]
$$

The constant term corresponds to the uniform distribution of negative charge that compensates the positive charge of the ions. And the remaining terms represent the oscillating charge density, screening the ions, which is directly related to the
Hartree potential $W^{H}(r)$ by Poisson's equation. By neglecting the terms of the expansion to the higher order than one, in the pseudo wave function, the oscillating electronic density $n^{\text {osc }}(\mathbf{r})$ reduces to:

$$
n^{o s c}(\mathbf{r})=\frac{1}{V} \sum_{k \leq k_{F}} \sum_{q \neq 0}\left[a_{q}(\mathbf{k}) \exp (i \mathbf{q} \cdot \mathbf{r})+a_{q}^{*}(\mathbf{k}) \exp (-i \mathbf{q} \cdot \mathbf{r})\right]=\frac{1}{V} \sum_{k \leq k_{F}} \sum_{q \neq 0} 2 a_{q}(\mathbf{k}) \exp (i \mathbf{q} \cdot \mathbf{r}),
$$

because $a_{q}^{*}(\mathbf{k}) \exp (-i \mathbf{q} \cdot \mathbf{r})=a_{q}(\mathbf{k}) \exp (i \mathbf{q} \cdot \mathbf{r})$ by the definition of $a_{q}(\mathbf{k})$.

As far as Poisson's equation $\nabla^{2} \Phi(\mathbf{r})=-\frac{e n^{o s c}(\mathbf{r})}{\varepsilon_{0}}$ is concerned, where $\Phi(\mathbf{r})$ is the electrostatic potential, it is rewritten with the Hartree potential $W^{H}(\mathbf{r})=e \Phi(\mathbf{r})$ as:

$$
\nabla^{2} W^{H}(\mathbf{r})=e \nabla^{2} \Phi(\mathbf{r})=-\frac{e^{2} n^{o s c}(\mathbf{r})}{\varepsilon_{0}} .
$$

To implement the method, it is easier to combine equations (111) and (112) after performing the standard transformations in $\mathbf{k}$-space ${ }^{11}$ :

$$
\begin{aligned}
& n^{o s c}(\mathbf{r})=\sum_{q \neq 0} n_{q}^{o s c} \exp (i \mathbf{q} \cdot \mathbf{r}) \\
& W^{H}(\mathbf{r})=\sum_{q \neq 0} W_{q}^{H} \exp (i \mathbf{q} \cdot \mathbf{r}) .
\end{aligned}
$$

By comparing equations (111) and (113), and by calculating the Laplacian of $W^{H}(\mathbf{r})$ with equation (114), we obtain the following relations:

$$
\begin{aligned}
n_{q}^{o s c} & =\frac{2}{V} \sum_{k \leq k_{F}} a_{q}(\mathbf{k}) \\
\nabla^{2} W^{H}(\mathbf{r}) & =\sum_{q \neq 0} W_{q}^{H}\left(-q^{2}\right) \exp (i \mathbf{q} \cdot \mathbf{r}) .
\end{aligned}
$$

The insertion of these expressions into equation (112) enables us to write the Poisson equation under the algebraic form:

$$
\begin{aligned}
\sum_{q \neq 0} W_{q}^{H}\left(-q^{2}\right) \exp (i \mathbf{q} \cdot \mathbf{r}) & =-\frac{e^{2}}{\varepsilon_{0}} \sum_{q \neq 0} n_{q}^{o s c} \exp (i \mathbf{q} \cdot \mathbf{r}), \\
W_{q}^{H}\left(-q^{2}\right) & =-\frac{e^{2}}{\varepsilon_{0}} n_{q}^{o s c} \\
W_{q}^{H} & =\frac{e^{2}}{\varepsilon_{0} q^{2}} n_{q}^{o s c} .
\end{aligned}
$$

\footnotetext{
${ }^{11}$ The transformation of the discrete sum into an integral over $q$ is done by using the formula:

$$
\sum_{q \neq 0} \rightarrow \frac{V}{(2 \pi)^{3}} \int d^{3} q
$$
}

This allows us to define the Fourier transform:

$$
n^{o s c}(\mathbf{r})=\sum_{q \neq 0} n_{q}^{o s c} \exp (i \mathbf{q r})=\frac{V}{(2 \pi)^{3}} \int n_{q}^{o s c} \exp (i \mathbf{q r}) d^{3} q
$$

The Hartree potential $W_{q}^{H}$ is then written explicitly in terms of the matrix element $|\langle\mathbf{k}+\mathbf{q}|W| \mathbf{k}\rangle|$, by using equations (115) and (109):

$$
W_{q}^{H}=\frac{e^{2}}{\varepsilon_{0} q^{2}} \frac{2}{V} \sum_{k \leq k_{F}} \frac{|\langle\mathbf{k}+\mathbf{q}|W| \mathbf{k}\rangle|}{\frac{\hbar^{2}}{2 m}\left[\mathbf{k}^{2}-|\mathbf{k}+\mathbf{q}|^{2}\right]},
$$

from which we arrive at the following final expression, by taking account of the formula given in footnote 9 :

$$
W_{q}^{H}=\frac{e^{2}}{2 \pi^{3} \varepsilon_{0} q^{2}} \int_{k \leq k_{F}} \frac{|\langle\mathbf{k}+\mathbf{q}|W| \mathbf{k}\rangle|}{\frac{\hbar^{2}}{2 m}\left[\mathbf{k}^{2}-|\mathbf{k}+\mathbf{q}|^{2}\right]} d^{3} k .
$$

\subsection{Concept of Dielectric Function}

In order to pursue the calculation of the Hartree potential (Eq. 119) without too much difficulty, assume that the pseudopotential is local. It therefore follows that the matrix element $|\langle\mathbf{k}+\mathbf{q}|W| \mathbf{k}\rangle|$ depends only on $q$ and can be extracted from the integral over $k$, which is not the case in the complete theory[23, 26, 27]. The calculation of $W_{q}^{H}$ is thus reduced to that of the integral:

$$
I=\int_{k \leq k_{F}} \frac{d^{3} k}{\frac{\hbar^{2}}{2 m}\left[\mathbf{k}^{2}-|\mathbf{k}+\mathbf{q}|^{2}\right]} .
$$

To achieve the integration over the states contained in the Fermi sphere, the sphere of radius $k_{F}$ is first drawn, then the transfer vector $\mathbf{q}$ is placed along the $z$-axis (Figure 7) and the following change of variables is performed:

$$
z=k_{F} \cos \theta
$$

with:

$$
n_{q}^{o s c}=\frac{1}{V} \int n^{o s c}(\mathbf{r}) \exp (-i \mathbf{q r}) d^{3} r
$$

It is the same for the function $W^{H}(\mathbf{r})$. 
so that the element of integration is the disc of volume:

$$
d^{3} k=\pi\left(k_{F}^{2}-z^{2}\right) d z .
$$

Moreover, as indicated by Hubbard[28], the integration merely depends on the values of the wave vector remote from those of the Fermi wave vector $\mathbf{k}_{F}$. This greatly simplifies the calculations because it is sufficient to integrate over the states $\mathbf{k} \simeq \mathbf{k}_{F}$, by setting:
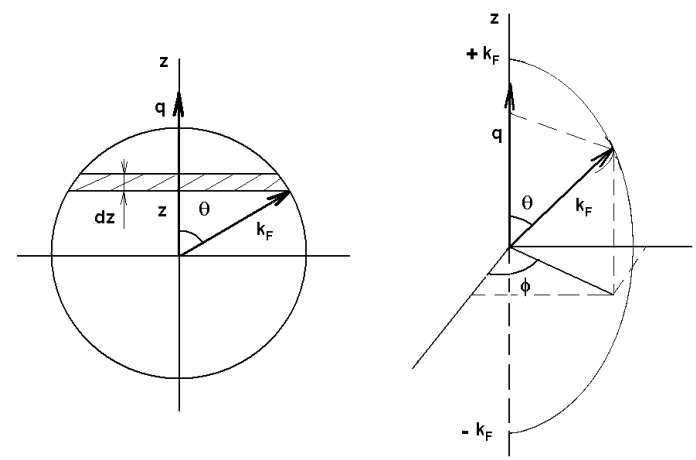

Figure 7. Choice of the integration variables for calculating the integral in equation (120), into the Fermi sphere. The shaded area corresponds to the volume element $d^{3} k$.

$$
\begin{aligned}
\mathbf{k}^{2}-|\mathbf{k}+\mathbf{q}|^{2} & \simeq \mathbf{k}_{F}^{2}-\left|\mathbf{k}_{F}+\mathbf{q}\right|^{2} \\
& =k_{F}^{2}-\left(k_{F}^{2}+2 q k_{F} \cos \theta+q^{2}\right) \\
& \simeq-q^{2}-2 q z .
\end{aligned}
$$

The integral $I$ reads:

$$
\begin{array}{r}
I=\frac{\pi m}{\hbar^{2} q} \int_{-k_{F}}^{+k_{F}} \frac{\left(z^{2}-k_{F}^{2}\right)}{\frac{q}{2}+z} d z, \quad \text { (124) } \begin{array}{l}
\text { pseudopotential and Hartree } \\
\text { Hartree potential is written in }
\end{array} \\
W_{q}^{H}=\left|\left\langle\mathbf{k}+\mathbf{q}\left|W^{H}\right| \mathbf{k}\right\rangle\right|=|\langle\mathbf{k}+\mathbf{q}|W| \mathbf{k}\rangle|-\left|\left\langle\mathbf{k}+\mathbf{q}\left|W_{0}\right| \mathbf{k}\right\rangle\right| .
\end{array}
$$

and its result reduces to the expression ${ }^{12}$ :

$$
I=\frac{\pi m}{\hbar^{2} q}\left[-q k_{F}+k_{F}^{2}\left(\frac{q^{2}}{4 k_{F}^{2}}-1\right) \ln \left|\frac{q+2 k_{F}}{q-2 k_{F}}\right|\right] .
$$

Further, the integral $I$ is simplified by putting $\eta=\frac{q}{k_{F}}$ (with $\eta<2)$ :

$$
I=-\frac{\pi m k_{F}}{\hbar^{2}}\left[1+\left(\frac{4-\eta^{2}}{4 \eta}\right) \ln \left|\frac{\eta+2}{\eta-2}\right|\right] .
$$

The substitution of equation (126) into equation (119) is then done to express the Hartree potential $W_{q}^{H}$ depending on the matrix element $|\langle\mathbf{k}+\mathbf{q}|W| \mathbf{k}\rangle|$ of the total pseudopotential as:

$$
\begin{aligned}
W_{q}^{H} & =\frac{e^{2}}{2 \pi^{3} \varepsilon_{0} q^{2}}|\langle\mathbf{k}+\mathbf{q}|W| \mathbf{k}\rangle| \\
& \times\left\{-\frac{\pi m k_{F}}{\hbar^{2}}\left[1+\left(\frac{4-\eta^{2}}{4 \eta}\right) \ln \left|\frac{\eta+2}{\eta-2}\right|\right]\right\} \\
& =\frac{e^{2}}{\varepsilon_{0} q^{2}}|\langle\mathbf{k}+\mathbf{q}|W| \mathbf{k}\rangle| X_{0}(q),
\end{aligned}
$$

where the Lindhard [29] function $X_{0}(q)$ defined by the following expression has been used:

$$
X_{0}(q)=-\frac{m k_{F}}{2 \pi^{2} \hbar^{2}}\left[1+\left(\frac{4-\eta^{2}}{4 \eta}\right) \ln \left|\frac{\eta+2}{\eta-2}\right|\right] .
$$

Thus, according to equation (127), the Hartree potential $W_{q}^{H}$ is related to the matrix element $|\langle\mathbf{k}+\mathbf{q}|W| \mathbf{k}\rangle|$ via the Lindhard function $X_{0}(q)$. But from the screening theory, the total pseudopotential is equal to the sum of the bare pseudopotential and Hartree potential (Eq. 64), so that the Hartree potential is written in $\mathbf{k}$-space as:

In eliminating $W_{q}^{H}$ between equations (127) and (129), the expression of the matrix element $|\langle\mathbf{k}+\mathbf{q}|W| \mathbf{k}\rangle|$ of the total pseudopotential is also expressed in terms of the matrix element $\left|\left\langle\mathbf{k}+\mathbf{q}\left|W_{0}\right| \mathbf{k}\right\rangle\right|$ as follows:

$$
|\langle\mathbf{k}+\mathbf{q}|W| \mathbf{k}\rangle|-\left|\left\langle\mathbf{k}+\mathbf{q}\left|W_{0}\right| \mathbf{k}\right\rangle\right|=\frac{e^{2}}{\varepsilon_{0} q^{2}}|\langle\mathbf{k}+\mathbf{q}|W| \mathbf{k}\rangle| X_{0}(q),
$$

hence:

$$
\begin{aligned}
& { }^{12} \text { Calculation of the integral: } \\
& J=\int_{-k_{F}}^{+k_{F}} \frac{\left(z^{2}-k_{F}^{2}\right)}{\frac{q}{2}+z} d z, \\
& =\int_{-k_{F}}^{+k_{F}} \frac{z^{2}-\frac{q^{2}}{4}}{\frac{q}{2}+z} d z+\left(\frac{q^{2}}{4}-k_{F}^{2}\right) \int_{-k_{F}}^{+k_{F}} \frac{1}{\frac{q}{2}+z} d z, \\
& =\left[\frac{z^{2}}{2}-\frac{q z}{2}\right]_{-k_{F}}^{+k_{F}}+\left(\frac{q^{2}}{4}-k_{F}^{2}\right) \ln \left|\frac{\frac{q}{2}+k_{F}}{\frac{q}{2}-k_{F}}\right| \text {, } \\
& =-q k_{F}+k_{F}^{2}\left(\frac{q^{2}}{4 k_{F}^{2}}-1\right) \ln \left|\frac{q+2 k_{F}}{q-2 k_{F}}\right| \text {. }
\end{aligned}
$$




$$
|\langle\mathbf{k}+\mathbf{q}|W| \mathbf{k}\rangle|=\frac{\left|\left\langle\mathbf{k}+\mathbf{q}\left|W_{0}\right| \mathbf{k}\right\rangle\right|}{\left(1-\frac{e^{2}}{\varepsilon_{0} q^{2}} X_{0}(q)\right)}=\frac{\left|\left\langle\mathbf{k}+\mathbf{q}\left|W_{0}\right| \mathbf{k}\right\rangle\right|}{\varepsilon^{H}(q)},
$$

where the Hartree dielectric function $\varepsilon^{H}(q)$ is defined by the expression:

$$
\varepsilon^{H}(q)=1-\frac{e^{2}}{\varepsilon_{0} q^{2}} X_{0}(q)=1+\frac{m e^{2}}{2 \pi^{2} \varepsilon_{0} \hbar^{2}} \frac{k_{F}}{q^{2}}\left[1+\left(\frac{4-\eta^{2}}{4 \eta}\right) \ln \left|\frac{\eta+2}{\eta-2}\right|\right] .
$$

This ends the screening theory that enables us to evaluate the electronic energy $E_{e l}$ by making possible the calculation of the matrix element $|\langle\mathbf{k}+\mathbf{q}|W| \mathbf{k}\rangle|$, which enters equation (98). It should be realized, in particular, that the form factor $|\langle\mathbf{k}+\mathbf{q}|w| \mathbf{k}\rangle|$ of the total pseudopotential, also known as the screened pseudopotential, is obtained by simple division of the form factor $\left|\left\langle\mathbf{k}+\mathbf{q}\left|w_{0}\right| \mathbf{k}\right\rangle\right|$ of the bare pseudopotential by the dielectric function $\varepsilon^{H}(q)$. Lastly, the expression of the form factor of the Ashcroft screened pseudopotential (Eq. 106) becomes:

$$
|\langle\mathbf{k}+\mathbf{q}|w| \mathbf{k}\rangle|=-\frac{Z e^{2}}{\varepsilon_{0} q^{2} \varepsilon^{H}(q)} \frac{N}{V} \cos q R_{c} .
$$

Incidentally, the Hartree potential $W_{q}^{H}$ can be also expressed in terms of the matrix element $\left|\left\langle\mathbf{k}+\mathbf{q}\left|W_{0}\right| \mathbf{k}\right\rangle\right|$, in using equations (129) and (131), under the form:

$$
W_{q}^{H}=\left[\frac{1}{\varepsilon^{H}(q)}-1\right]\left|\left\langle\mathbf{k}+\mathbf{q}\left|W_{0}\right| \mathbf{k}\right\rangle\right| .
$$

As an indication, the bare and screened form factors as well as the dielectric function are depicted in figure 8 .

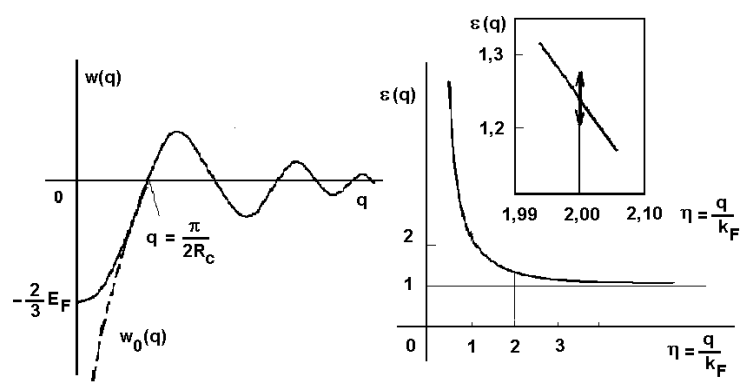

Figure 8. Schematic representations of the bare form factor $w_{0}(q)$ and the screened form factor $w(q)$, as well as the Hartree dielectric function $\varepsilon(q)$.

It should be mentioned that the curves representing the functions $w(q)$ and $w_{0}(q)$ differ only for values $q<\frac{\pi}{2 R_{c}}$. The mathematical study of these functions poses no difficulty. The salient characteristics of the curve $w(q)=|\langle\mathbf{k}+\mathbf{q}|w| \mathbf{k}\rangle|$ are the damped oscillations, the value of the first node, at $q=\frac{\pi}{2 R_{c}}$, and the value at the origin that is $-\frac{2}{3} \frac{\hbar^{2} k_{F}^{2}}{2 m}$. This value is found by calculating the limit of $|\langle\mathbf{k}+\mathbf{q}|w| \mathbf{k}\rangle|$ at $q=0$. It is easily

obtained by using the relation $\frac{N Z}{V}=\frac{k_{F}^{3}}{3 \pi^{2}}$ (see footnote 9 ) and by looking at the behavior of the dielectric function $\varepsilon^{H}(q)$. The latter is a decreasing function varying between the limits:

$$
\begin{aligned}
\varepsilon^{H}(q) & \sim 1+\frac{m e^{2}}{\pi^{2} \varepsilon_{0} \hbar^{2}} \frac{k_{F}}{q^{2}} \quad \text { when } \quad q \rightarrow 0, \\
& \sim 1+\frac{4}{3} \frac{m e^{2}}{\pi^{2} \varepsilon_{0} \hbar^{2}} \frac{k_{F}^{3}}{q^{4}} \quad \text { when } \quad q \rightarrow \infty .
\end{aligned}
$$

But its most striking feature is the logarithmic singularity at $q=2 k_{F}$. Indeed, it can be verified that ${ }^{13}$ :

$$
\varepsilon^{H}\left(2 k_{F}\right)=1+\frac{m e^{2}}{8 \pi^{2} \varepsilon_{0} \hbar^{2}} \frac{1}{k_{F}} \times\left[2-2 \sum_{p \geq 1}^{\infty} \frac{1}{(2 p-1)(2 p+1)}\right]=1+\frac{m e^{2}}{8 \pi^{2} \varepsilon_{0} \hbar^{2}} \frac{1}{k_{F}},
$$

${ }^{13}$ The calculation of the limits of the functions $\varepsilon^{H}(q)$ and $\frac{d \varepsilon^{H}(q)}{d \varepsilon}$, at $q=$ Products, Acad. Press, New York (1965), pages 3 and 8]. $2 k_{F}$, makes use of the series expansions:

$$
\text { and } \begin{aligned}
\sum_{p \geq 1}^{\infty} \frac{1}{(2 p-1)(2 p+1)} & =\frac{1}{2}, \\
\sum_{p \geq 1}^{\infty} \frac{1}{(2 p-1)} & \rightarrow \infty,
\end{aligned}
$$

[see I. S. Gradshteyn and I. M. Ryzhik, Table of Integrals, Series and 


$$
\left(\frac{d \varepsilon^{H}}{d q}\right)_{2 k_{F}}=-\frac{m e^{2}}{8 \pi^{2} \varepsilon_{0} \hbar^{2}} \frac{1}{k_{F}}\left[1+\sum_{p \geq 1}^{\infty} \frac{1}{(2 p-1)}\right] \rightarrow-\infty .
$$

This singularity is not visible to the naked eye on the curve, but nevertheless it is the source of the oscillations of interionic potential in liquid metals.

\section{Interionic Potentials in Liquid Metals}

With the matrix element $|\langle\mathbf{k}+\mathbf{q}|W| \mathbf{k}\rangle|$ (Eq. 131) in hand, we are able to calculate the electronic energy $E_{e l}$ defined by equation (98). Given the simplifications recommended in the screening theory, the expression of $E_{e l}$ is:

$$
E_{e l}=\frac{2 V}{(2 \pi)^{3}} \int_{k \leq k_{F}} \frac{\hbar^{2} k^{2}}{2 m} d^{3} k+\frac{2 V}{(2 \pi)^{3}} \int_{k \leq k_{F}}\langle\mathbf{k}|W| \mathbf{k}\rangle d^{3} k+\sum_{q \neq 0} \frac{2 V}{(2 \pi)^{3}}|\langle\mathbf{k}+\mathbf{q}|W| \mathbf{k}\rangle|^{2} \int_{k \leq k_{F}} \frac{1}{\frac{\hbar^{2}}{2 m}\left[\mathbf{k}^{2}-|\mathbf{k}+\mathbf{q}|^{2}\right]} d^{3} k .
$$

It is easy to check that the first term on the right hand side corresponds to the Fermi energy $E_{e l}^{(1)}=N Z E_{F}$ of the free electron gas, with $E_{F}=\frac{3}{5} \frac{\hbar^{2} k_{F}^{2}}{2 m}$ (Eq. 68). The second term on the RHS represents the average energy $E_{e l}^{(2)}=\overline{\langle\mathbf{k}|W| \mathbf{k}\rangle}$.
As regards the third term, $E_{e l}^{(3)}$, it is much more interesting because, unlike the first two terms, it depends on the ionic configuration and it is at the origin of the calculation of the interionic potential. Let us consider it carefully. With equation (119) that is recalled below:

$$
W_{q}^{H}=\frac{e^{2}}{2 \pi^{3} \varepsilon_{0} q^{2}}|\langle\mathbf{k}+\mathbf{q}|W| \mathbf{k}\rangle| \int_{k \leq k_{F}} \frac{1}{\frac{\hbar^{2}}{2 m}\left[\mathbf{k}^{2}-|\mathbf{k}+\mathbf{q}|^{2}\right]} d^{3} k,
$$

the term $E_{e l}^{(3)}$ turns into:

$$
E_{e l}^{(3)}=\sum_{q \neq 0} \frac{2 V}{(2 \pi)^{3}}|\langle\mathbf{k}+\mathbf{q}|W| \mathbf{k}\rangle| W_{q}^{H} \frac{2 \pi^{3} \varepsilon_{0} q^{2}}{e^{2}} .
$$

However, when use is made of equations (131) and (134), repeated below:

$$
|\langle\mathbf{k}+\mathbf{q}|W| \mathbf{k}\rangle|=\frac{\left|\left\langle\mathbf{k}+\mathbf{q}\left|W_{0}\right| \mathbf{k}\right\rangle\right|}{\varepsilon^{H}(q)},
$$

and

$$
W_{q}^{H}=\left[\frac{1}{\varepsilon^{H}(q)}-1\right]\left|\left\langle\mathbf{k}+\mathbf{q}\left|W_{0}\right| \mathbf{k}\right\rangle\right|
$$

the contribution $E_{e l}^{(3)}$ can also be written as a function of the matrix element $\left|\left\langle\mathbf{k}+\mathbf{q}\left|W_{0}\right| \mathbf{k}\right\rangle\right|$ of the bare pseudopotential and the dielectric function $\varepsilon^{H}(q)$ :

$$
E_{e l}^{(3)}=\sum_{q \neq 0} \frac{2 V}{(2 \pi)^{3}} \frac{\left|\left\langle\mathbf{k}+\mathbf{q}\left|W_{0}\right| \mathbf{k}\right\rangle\right|^{2}}{\varepsilon^{H}(q)}\left[\frac{1}{\varepsilon^{H}(q)}-1\right] \frac{2 \pi^{3} \varepsilon_{0} q^{2}}{e^{2}}=\frac{\varepsilon_{0} V}{2 e^{2}} \sum_{q \neq 0} q^{2} \frac{\left|\left\langle\mathbf{k}+\mathbf{q}\left|W_{0}\right| \mathbf{k}\right\rangle\right|^{2}}{\varepsilon^{H}(q)}\left[\frac{1}{\varepsilon^{H}(q)}-1\right] .
$$

It should be recalled that the electronic energy $E_{e l}$ includes the electron-electron energy as well as the electron-ion energy. In the Hartree-Fock method, which has been used to calculate $E_{e l}=\sum_{i} E_{i}$, the energy of each electron (Eq. 61) is calculated in the field of ions and all other electrons. Therefore, the interaction energy between each pair of electrons $(i, j)$ is counted twice, once when the electron $i$ is at the origin and once when the electron $j$ is at the origin. Thus, the electron-electron interaction $E_{e l-e l}$ must be subtracted from the electronic energy $E_{e l}$ (see footnote 10).
In view of this, $E_{e l-e l}$ is evaluated in terms of the Hartree potential $W^{H}(r)$ and oscillating electronic density $n^{o s c}(r)$ by the formula:

$$
E_{e l-e l}=\frac{1}{2} \int n^{o s c}(r) W^{H}(r) d^{3} r .
$$

But, as in the case of the calculation of $E_{e l}$, it is preferable to express the interaction energy $E_{e l-e l}$ in $\mathbf{k}$-space by using equations (113) and (114). After transforming $n^{o s c}(r)$ and 
$W^{H}(r), E_{e l-e l}$ reads $^{14}$ :

$$
E_{e l-e l}=\frac{V}{2} \sum_{q \neq 0} n_{q}^{o s c} W_{q}^{* H}
$$

Then, taking account of equation (117), we replace $n_{q}^{\text {osc }}$ as a function of $W_{q}^{H}$ and rewrite the interaction energy $E_{e l-e l}$ as:

$$
E_{e l-e l}=\frac{\varepsilon_{0} V}{2 e^{2}} \sum_{q \neq 0} q^{2} W_{q}^{H} W_{q}^{* H}
$$

Finally, equation (134) is employed to express the interaction energy $E_{e l-e l}$ in terms of the matrix element $\left|\left\langle\mathbf{k}+\mathbf{q}\left|W_{0}\right| \mathbf{k}\right\rangle\right|$ and dielectric function $\varepsilon^{H}(q)$ as:

$$
E_{e l-e l}=\frac{\varepsilon_{0} V}{2 e^{2}} \sum_{q \neq 0} q^{2}\left[\frac{1}{\varepsilon^{H}(q)}-1\right]^{2}\left|\left\langle\mathbf{k}+\mathbf{q}\left|W_{0}\right| \mathbf{k}\right\rangle\right|^{2} .
$$

This expression represents the electron-electron energy to be subtracted from the electronic energy $E_{e l}$. But the full calculation of the energy of liquid metals requires that the ion-ion energy $E_{i o-i o}$, defined by equation (53), is added to $\left(E_{e l}-E_{e l-e l}\right)$. The combination of all these terms leads to the expression of the energy of liquid metals:

$$
\begin{aligned}
E & =E_{e l}^{(1)}+E_{e l}^{(2)}+E_{e l}^{(3)}-E_{e l-e l}+E_{i o-i o}=N Z E_{F}+\overline{\langle\mathbf{k}|W| \mathbf{k}\rangle}+\frac{\varepsilon_{0} V}{2 e^{2}} \sum_{q \neq 0} q^{2} \frac{\left|\left\langle\mathbf{k}+\mathbf{q}\left|W_{0}\right| \mathbf{k}\right\rangle\right|^{2}}{\varepsilon^{H}(q)}\left[\frac{1}{\varepsilon^{H}(q)}-1\right] \\
& -\frac{\varepsilon_{0} V}{2 e^{2}} \sum_{q \neq 0} q^{2}\left[\frac{1}{\varepsilon^{H}(q)}-1\right]^{2}\left|\left\langle\mathbf{k}+\mathbf{q}\left|W_{0}\right| \mathbf{k}\right\rangle\right|^{2}+\frac{1}{2} \sum_{\alpha, \beta \neq \alpha} \frac{1}{4 \pi \varepsilon_{0}} \frac{Z^{2} e^{2}}{\left|\mathbf{R}_{\alpha}-\mathbf{R}_{\beta}\right|}
\end{aligned}
$$

or still, in the simplified form:

$$
E=N Z E_{F}+\overline{\langle\mathbf{k}|W| \mathbf{k}\rangle}+\frac{\varepsilon_{0} V}{2 e^{2}} \sum_{q \neq 0} q^{2}\left|\left\langle\mathbf{k}+\mathbf{q}\left|W_{0}\right| \mathbf{k}\right\rangle\right|^{2}\left[\frac{1}{\varepsilon^{H}(q)}-1\right]+\frac{1}{2} \sum_{\alpha, \beta \neq \alpha} \frac{1}{4 \pi \varepsilon_{0}} \frac{Z^{2} e^{2}}{\left|\mathbf{R}_{\alpha}-\mathbf{R}_{\beta}\right|} .
$$

This is the expression for the total energy of liquid metals identified in equation (47). Before comparing equations (47) and (148), we transform the third term on the right hand side of equation (148) with the intention of splitting off the energy contribution depending on the ionic configuration from that which does not depend on it. To do this, the matrix element is separated by using equation (95):

$$
\left|\left\langle\mathbf{k}+\mathbf{q}\left|W_{0}\right| \mathbf{k}\right\rangle\right|^{2}=\frac{1}{N^{2}} \sum_{\alpha, \beta} \exp \left[-i \mathbf{q} \cdot\left(\mathbf{R}_{\beta}-\mathbf{R}_{\alpha}\right)\right] \times\left|\left\langle\mathbf{k}+\mathbf{q}\left|w_{0}\right| \mathbf{k}\right\rangle\right|^{2}
$$

This relation allows writing the third term of equation (148) in the following manner:

$$
\frac{\varepsilon_{0} V}{2 e^{2}} \sum_{q \neq 0} q^{2}\left|\left\langle\mathbf{k}+\mathbf{q}\left|W_{0}\right| \mathbf{k}\right\rangle\right|^{2}\left[\frac{1}{\varepsilon^{H}(q)}-1\right]=\sum_{q \neq 0}\left\{\frac{1}{N} \sum_{\alpha, \beta \neq \alpha} \exp \left[-i \mathbf{q} \cdot\left(\mathbf{R}_{\beta}-\mathbf{R}_{\alpha}\right)\right] F(q)+\frac{1}{N} \sum_{\alpha=\beta} \exp \left[-i \mathbf{q} \cdot\left(\mathbf{R}_{\beta}-\mathbf{R}_{\alpha}\right)\right] F(q)\right\}
$$

as a function of the energy wave number characteristic:

$$
F(q)=-\frac{\varepsilon_{0} V}{2 e^{2} N} q^{2}\left|\left\langle\mathbf{k}+\mathbf{q}\left|w_{0}\right| \mathbf{k}\right\rangle\right|^{2}\left[1-\frac{1}{\varepsilon^{H}(q)}\right] .
$$

Because $\sum_{\alpha=\beta} \exp \left[-i \mathbf{q} \cdot\left(\mathbf{R}_{\beta}-\mathbf{R}_{\alpha}\right)\right]=N$, the second term in braces of equation (150) is equal to $F(q)$. As it does not depend on the ion configuration, it may be gathered with the terms $E_{e l}^{(1)}$ and $E_{e l}^{(2)}$ to form the contribution to the energy of liquid metals $N u_{g}(V, T)$, which is only a function of the volume and temperature. By contrast, the first term in braces of equation ( 150) plus the ion-ion interaction $E_{i o-i o}$ provide together the contribution to the energy of liquid metal $U_{N}\left(V, T, \mathbf{R}_{1}, \ldots, \mathbf{R}_{N}\right)=$

\footnotetext{
${ }^{14}$ The calculation of the interaction energy $E_{e l-e l}$ is done as follows:

$$
\begin{aligned}
E_{e l-e l} & =\frac{1}{2} \int \sum_{q \neq 0} n_{q}^{o s c} \exp (i \mathbf{q r}) W^{H}(r) d^{3} r, \\
& =\frac{V}{2} \sum_{q \neq 0} n_{q}^{o s c} \frac{1}{V} \int \exp (i \mathbf{q r}) W^{H}(r) d^{3} r, \\
& =\frac{V}{2} \sum_{q \neq 0} n_{q}^{o s c} W_{-q}^{H}=\frac{V}{2} \sum_{q \neq 0} n_{q}^{o s c} W_{q}^{* H} .
\end{aligned}
$$
}


$\frac{1}{2} \sum_{\alpha, \beta \neq \alpha} u\left(R_{\alpha \beta}\right)$, which depends on the volume, temperature and configuration (Eq. 48). This last contribution takes the form:

$$
\frac{1}{2} \sum_{\alpha, \beta \neq \alpha} u\left(R_{\alpha \beta}\right)=\frac{1}{N} \sum_{\alpha, \beta \neq \alpha} \sum_{q \neq 0} \exp \left[-i \mathbf{q} \cdot\left(\mathbf{R}_{\beta}-\mathbf{R}_{\alpha}\right)\right] F(q)+\frac{1}{2} \sum_{\alpha, \beta \neq \alpha} \frac{1}{4 \pi \varepsilon_{0}} \frac{Z^{2} e^{2}}{\left|\mathbf{R}_{\alpha}-\mathbf{R}_{\beta}\right|} .
$$

To evaluate this energy contribution, we replace the discrete sum over $q$ by an integral ${ }^{15}$ and take $\mathbf{R}_{\beta}-\mathbf{R}_{\alpha}=\mathbf{r}_{\alpha \beta}$ for convenience:

$$
\frac{1}{2} \sum_{\alpha, \beta \neq \alpha} u \quad\left(r_{\alpha \beta}\right)=\frac{1}{2} \sum_{\alpha, \beta \neq \alpha}\left[\frac{1}{4 \pi \varepsilon_{0}} \frac{Z^{2} e^{2}}{r_{\alpha \beta}}+\frac{2 V}{(2 \pi)^{3} N} \int F(q) \exp \left(-i \mathbf{q} \cdot \mathbf{r}_{\alpha \beta}\right) d^{3} q\right] .
$$

This is the equation required for calculating the interionic potential in liquid metals and whose the formal expression is:

$$
u(r)=\frac{1}{4 \pi \varepsilon_{0}} \frac{Z^{2} e^{2}}{r}+\frac{2 V}{(2 \pi)^{3} N} \int F(q) \exp (-i \mathbf{q} \cdot \mathbf{r}) d^{3} q .
$$

The interionic potential $u(r)$ consists of two terms. The first one is the direct interaction between the ions, $u_{\text {dir }}(r)$, and the second one the interaction induced by the electron gas, $u_{\text {ind }}(r)$. The latter contribution depends explicitly on the bare form factor $\left\langle\mathbf{k}+\mathbf{q}\left|w_{0}\right| \mathbf{k}\right\rangle$ and on the dielectric function $\varepsilon^{H}(q)$ through the energy wave number characteristic $F(q)$. It should be noted the great interest introduced by the function $F(q)$ as a measure of the induced interaction, $u_{\text {ind }}(r)$, since $u_{\text {ind }}(r)$ is nothing else than the Fourier transform of the energy wave number characteristic $F(q)$ :

$$
u_{\text {ind }}(r)=F T\left[\frac{2 V}{N} F(q)\right] \text {. }
$$

Some interionic potentials in liquid metals have been portrayed in figure 9 .

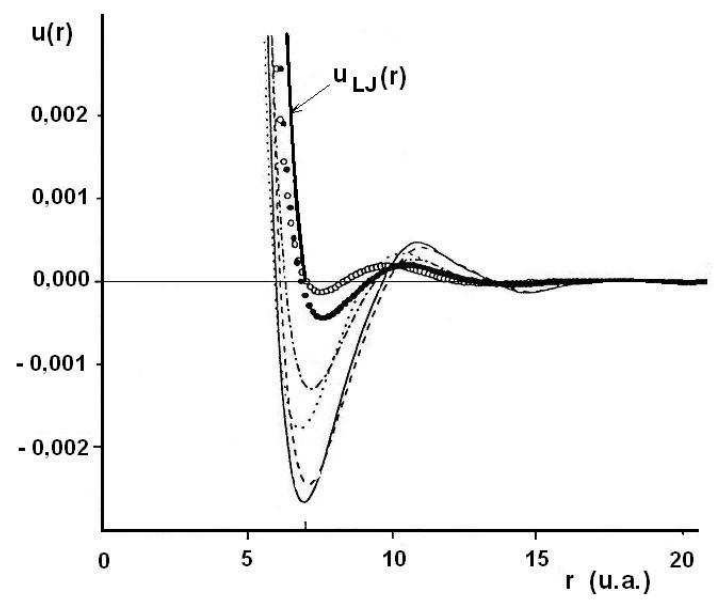

Figure 9. Interionic potential of sodium calculated with different pseudopotentials. The repulsive part of the Lennard-Jones potential is drawn in thick lines for comparison.

${ }^{15}$ The calculation of the discrete sum over $q$ is done using the formula:

$$
\sum_{q \neq 0} \rightarrow \frac{V}{(2 \pi)^{3}} \int d^{3} q
$$

It is remarkable that they have attractive wells varying significantly from one to another. Moreover, they possess an oscillating long-range part, called the Friedel oscillations[30], which is due to the logarithmic singularity of the dielectric function at $q=2 k_{F}$. Concerning the repulsive part at short range, it is softer than that of the liquid rare gases for which the valence electron shells are completely filled.

\section{Linear Response: Corrections for Exchange and Correlation}

It has been mentioned that the calculation of the energy of liquid metals is done by adding to the configurational energy $\frac{1}{2} \sum_{\alpha, \beta \neq \alpha} u\left(r_{\alpha \beta}\right)$, the term $N u_{g}(V, T)$ depending only on the volume and temperature. By consulting the literature[31, 32, $33]$, it can be seen that $N u_{g}(V, T)$ contains, in addition to the Fermi energy, the terms coming from the exchange and correlation effects in the electron gas. These effects also affect significantly the interionic potential by modifying the Hartree dielectric function.

To introduce the exchange and correlation effects, the response function theory is usually used which is an alternative to the screening theory[34, 35, 36]. In the following, we present the outlines of this theory by looking at how the free electron gas responds when it is subjected to the perturbing potential $W_{0}(q)$ generated by the ions. If $W_{0}(q)$ is sufficiently small, the linear response theory states that the oscillating part $n^{o s c}(q)$ of the induced charge density is linearly related to $W_{0}(q)$ by the relation:

$$
n^{o s c}(q)=\chi^{(1)}(q) W_{0}(q),
$$

where $\chi^{(1)}(q)$ is the linear response function of the perturbed system. Do not confuse this equation with equation (117) that connects the oscillating charge density $n^{\text {osc }}(q)$ with the Hartree potential $W^{H}(q)$.

\subsection{Without Exchange and Correlation Effects}

In that case the total potential $W(q)$ resulting from a hypothetical test charge (electronic or non-electronic) is equal 
to (Eqs. 129 and 131):

$$
W(q)=W_{0}(q)+W^{H}(q)=\frac{W_{0}(q)}{\varepsilon^{H}(q)},
$$

where the Hartree potential $W^{H}(q)$ represents the energy of electron-electron interaction. After replacing $W^{H}(q)$ by its expression taken from equation (117), and with the help of equation (156), the previous relation is written as:

$$
\begin{aligned}
W(q) & =W_{0}(q)+\frac{e^{2}}{\varepsilon_{0} q^{2}} n^{o s c}(q)=\frac{W_{0}(q)}{\varepsilon^{H}(q)} \\
& =W_{0}(q)+\frac{e^{2}}{\varepsilon_{0} q^{2}} \chi^{(1)}(q) W_{0}(q) \\
& =\frac{W_{0}(q)}{\varepsilon^{H}(q)} .
\end{aligned}
$$

This expression is used to link directly the response function $\chi^{(1)}(q)$ to the Hartree dielectric function $\varepsilon^{H}(q)$ by the following relation:

$$
\frac{1}{\varepsilon^{H}(q)}=1+\frac{e^{2}}{\varepsilon_{0} q^{2}} \chi^{(1)}(q)
$$

or

$$
\chi^{(1)}(q)=\frac{\varepsilon_{0} q^{2}}{e^{2}}\left[\frac{1}{\varepsilon^{H}(q)}-1\right] .
$$

It should be noted that, in the general formalism, the linear response function $\chi^{(1)}(q)$ is related to the static structure factor of the perturbed system. Besides, in the case where the perturbed system is a system containing pseudoions embedded into the electron gas, the response function can be determined within the framework of the theory of the one component plasma[37, 38, 39].

By taking advantage of the results of the screening theory and after extracting the quantity $\frac{\varepsilon_{0} q^{2}}{e^{2}}$ from equations (160) and (132), the response function $\chi^{(1)}(q)$ may be expressed by means of the Lindhard function $X_{0}(q)$ as:

$$
\begin{gathered}
\chi^{(1)}(q)=\frac{X_{0}(q)}{\varepsilon^{H}(q)}, \quad(161) \quad \begin{array}{l}
\text { It has been established that the local field } \\
\text { equation }(157) \text { by reducing the screening } \\
\text { relation: }
\end{array} \\
W^{\text {mod }}(q)=W_{0}(q)+W^{H}(q)+W^{x c}(q)=W_{0}(q)+\frac{e^{2}}{\varepsilon_{0} q^{2}} n_{x c}^{o s c}(q)-\frac{e^{2}}{\varepsilon_{0} q^{2}} n_{x c}^{o s c}(q) G(q) .
\end{gathered}
$$

that permits rewriting equation (156) as:

$$
n^{o s c}(q)=\frac{X_{0}(q)}{\varepsilon^{H}(q)} W_{0}(q)=X_{0}(q) W(q) .
$$
response function (Eq. 160), namely:

$$
F(q)=\frac{V}{2 N} \chi^{(1)}(q)\left|\left\langle\mathbf{k}+\mathbf{q}\left|w_{0}\right| \mathbf{k}\right\rangle\right|^{2},
$$
gas, is written in the compact form:

\subsection{With Exchange and Correlation Effects} equation (162) as:

$$
n_{x c}^{o s c}(q)=X_{0}(q) W^{\bmod }(q),
$$
each other for large values of $q$.

The response function $\chi^{(1)}(q)$ is a very important quantity, which measures the ability of the free electron gas to respond to a perturbation and allows the calculation of the indirect part of the interionic potential, $u_{i n d}(r)$. To show it, we express the energy wave number characteristic (Eq. 151) in terms of the

and substitute equation (163) in equation (155), so that the contribution of the interionic potential, induced by the electron

$$
u_{\text {ind }}(r)=F T\left[\left(\frac{V}{N}\right)^{2} \chi^{(1)}(q)\left|\left\langle\mathbf{k}+\mathbf{q}\left|w_{0}\right| \mathbf{k}\right\rangle\right|^{2}\right] .
$$

Here the perturbing potential $W_{0}(q)$ induces an oscillating charge density $n_{x c}^{o s c}(q)$ that differs from $n^{o s c}(q)$ and modifies

where $X_{0}(q)$ is the Lindhard function and $W^{\bmod }(q)$ the modified total potential acting on a hypothetical electronic test charge. This potential contains, in addition to the terms $W_{0}(q)$ and $W^{H}(q)=\frac{e^{2}}{\varepsilon_{0} q^{2}} n_{x c}^{o s c}(q)$, a local field correction $W^{x c}(q)=-\frac{e^{2}}{\varepsilon_{0} q^{2}} n_{x c}^{o s c}(q) G(q)$, where the local field function $G(q)$ exists in different analytical forms[40, 41, 42], which are discussed elsewhere[43]. Simply note that they vary with $q^{2}$ for small values of $q$, and have very different behavior from

It has been established that the local field correction alters equation (157) by reducing the screening according to the

By using equation (165) to substitute $n_{x c}^{o s c}(q)$ in the above relation, this yields:

$$
W^{\mathrm{mod}}(q)=W_{0}(q)+\frac{e^{2}}{\varepsilon_{0} q^{2}} X_{0}(q) W^{\mathrm{mod}}(q)[1-G(q)]=\frac{W_{0}(q)}{1-\frac{e^{2}}{\varepsilon_{0} q^{2}} X_{0}(q)[1-G(q)]}=\frac{W_{0}(q)}{\varepsilon(q)}
$$

where the modified dielectric function $\varepsilon(q)$ is defined by the expression:

$$
\varepsilon(q)=1-\frac{e^{2}}{\varepsilon_{0} q^{2}} X_{0}(q)[1-G(q)] .
$$

But, with equation (132), the above relation can also be written in the simple form:

$$
\varepsilon(q)=1+\left[\varepsilon^{H}(q)-1\right][1-G(q)] .
$$

It should be stressed that an ion (hypothetical non-electronic test charge) immersed in the electron gas feels the potential $\left[W_{0}(q)+W^{H}(q)\right]$ and not $\left[W_{0}(q)+W^{H}(q)+W^{x c}(q)\right]$. 
Therefore, in calculating the energy wave number characteristic with equation (151), the Hartree dielectric function $\varepsilon^{H}(q)$ must be replaced, not by the dielectric function $\varepsilon(q)$, but by the new dielectric function $\varepsilon_{i o}(q)$ defined with the following relation, similar to equation (158):

$$
W_{0}(q)+W^{H}(q)=W_{0}(q)+\frac{e^{2}}{\varepsilon_{0} q^{2}} n_{x c}^{o s c}(q)=\frac{W_{0}(q)}{\varepsilon_{i o}(q)} .
$$

To find the expression of $\varepsilon_{i o}(q)$ as a function of $\varepsilon^{H}(q)$ and $G(q)$, just jointly use equations (165) and (167) to replace $n_{x c}^{o s c}(q)$ in the previous relation, that is to say:

$$
W_{0}(q)+\frac{e^{2}}{\varepsilon_{0} q^{2}} X_{0}(q) \frac{W_{0}(q)}{\varepsilon(q)}=\frac{W_{0}(q)}{\varepsilon_{i o}(q)},
$$

from which $\varepsilon_{i o}(q)$ may be extracted:

$$
\varepsilon_{i o}(q)=\frac{1}{1+\frac{e^{2}}{\varepsilon_{0} q^{2}} X_{0}(q) \frac{1}{\varepsilon(q)}}
$$

Now, if we use equation (168) to eliminate the quantity $\frac{e^{2}}{\varepsilon_{0} q^{2}} X_{0}(q)$, we obtain the simple relationship:

$$
\left[1-\frac{1}{\varepsilon_{i o}(q)}\right]=\left[1-\frac{1}{\varepsilon(q)}\right] \frac{1}{[1-G(q)]} .
$$

It is thus clear that the dielectric function $\varepsilon_{i o}(q)$ depends on the local field function $G(q)$ and the modified dielectric function $\varepsilon(q)$, which itself depends on $G(q)$ and the Lindhard function $X_{0}(q)$ (Eq. 168).

As a result, if the effects of exchange and correlation are taken into account, the interionic potential $u(r)$ can be still calculated with the energy wave number characteristic $F(q)$, by using equation (154), in which the Hartree dielectric function is replaced by the dielectric function $\varepsilon_{i o}(q)$ :

$$
F(q)=-\frac{\varepsilon_{0} V}{2 e^{2} N} q^{2}\left|\left\langle\mathbf{k}+\mathbf{q}\left|w_{0}\right| \mathbf{k}\right\rangle\right|^{2}\left[1-\frac{1}{\varepsilon_{i o}(q)}\right] .
$$

To end this discussion, note that the interionic potential $u(r)$ can also be written as a simple integral over $q$ after a number of changes. The first change consists in the introduction of the normalized energy wave number characteristic $F_{N}(q)$, equal to unity at $q=0$. The latter is performed by calculating the limit of equation (174) when $q=0$, and by noticing that $\lim _{q \rightarrow 0}$ $\varepsilon_{i o}(q) \rightarrow \infty$ and $\lim _{q \rightarrow 0}\left\langle\mathbf{k}+\mathbf{q}\left|w_{0}\right| \mathbf{k}\right\rangle \sim-\frac{Z e^{2}}{\varepsilon_{0}} \frac{N}{V} \frac{1}{q^{2}}$ (Eq. 106). It results that:

$$
F_{N}(q)=-\frac{2 \varepsilon_{0}}{Z^{2} e^{2}} \frac{V}{N} q^{2} F(q)
$$

The second change is made by reducing the triple integral in equation (154) to a simple integral ${ }^{16}$ that modifies $u(r)$ as follows:

$$
u(r)=\frac{1}{4 \pi \varepsilon_{0}} \frac{Z^{2} e^{2}}{r}-\frac{2 V}{(2 \pi)^{3} N} 4 \pi \int_{0}^{\infty}\left[\frac{Z^{2} e^{2}}{2 \varepsilon_{0}} \frac{N}{V} \frac{1}{q^{2}} F_{N}(q)\right] \times \frac{\sin (q r)}{q r} q^{2} d q .
$$

The third change comes from to writing the direct interaction between the ions in $\mathrm{k}$-space, before performing the following simplifications ${ }^{17}$ :

$$
\begin{aligned}
u(r) & =\frac{1}{4 \pi \varepsilon_{0}} \frac{Z^{2} e^{2}}{r} \frac{2}{\pi} \int_{0}^{\infty} \frac{\sin (q r)}{q r} r d q-\frac{2 V}{(2 \pi)^{3} N} \times 4 \pi \int_{0}^{\infty}\left[\frac{Z^{2} e^{2}}{2 \varepsilon_{0}} \frac{N}{V} \frac{1}{q^{2}} F_{N}(q)\right] \frac{\sin (q r)}{q r} q^{2} d q \\
& =\frac{Z^{2} e^{2}}{2 \pi^{2} \varepsilon_{0}} \int_{0}^{\infty} \frac{\sin (q r)}{q r} d q-\frac{Z^{2} e^{2}}{2 \pi^{2} \varepsilon_{0}} \int_{0}^{\infty} F_{N}(q) \frac{\sin (q r)}{q r} d q=\frac{Z^{2} e^{2}}{2 \pi^{2} \varepsilon_{0}} \int_{0}^{\infty}\left[1-F_{N}(q)\right] \frac{\sin (q r)}{q r} d q
\end{aligned}
$$

Finally, the interionic potential $u(r)$ is still written in the more compact form:

$$
u(r)=\frac{Z^{2} e^{2}}{8 \pi^{3} \varepsilon_{0}} \int\left[\frac{1-F_{N}(q)}{q^{2}}\right] \exp (-i \mathbf{q} \cdot \mathbf{r}) d \mathbf{q}
$$

which, compared with its Fourier transform

$$
u(r)=\frac{1}{8 \pi^{3}} \int u(q) \exp (-i \mathbf{q} \cdot \mathbf{r}) d \mathbf{q}
$$

provides a particularly simple relation between $u(q)$ and

$$
\begin{aligned}
& { }^{16} \text { With the changes of variables } d^{3} q=q^{2} \sin \theta d \theta d \varphi d q \text { and } \mathbf{q r}=q r \mu, \\
& \text { where } \mu=\cos \theta \text {, the integral is simplified to (Eq. 104): } \\
& \begin{aligned}
\int F(q) \exp (-i \mathbf{q} \cdot \mathbf{r}) d^{3} q & =2 \pi \int_{0}^{\infty} F(q) \frac{1}{i q r}[\exp (-i q r)]_{+1}^{-1} q^{2} d q, \\
& =4 \pi \int_{0}^{\infty} F(q) \frac{\sin (q r)}{q r} q^{2} d q .
\end{aligned}
\end{aligned}
$$

${ }^{17}$ For this, the use is made of the result:

$$
\int_{0}^{\infty} \frac{\sin (q r)}{q r} r d q=\frac{\pi}{2}
$$


$F_{N}(q)$, namely:

$$
u(q)=\frac{Z^{2} e^{2}}{\varepsilon_{0} q^{2}}\left[1-F_{N}(q)\right] .
$$

\section{Concluding Remarks}

In the rare gas liquids, in which the atoms are characterized by closed electronic shells, the interatomic potentials are typified by a strong repulsion at short distances, to prevent collapse of the substance, and an attractive tail to ensure the cohesion. By contrast, in the normal metals where the valence bands are very well separated from the next lowest core-level bands, the interionic potentials exhibit a repulsion in the core and a long range oscillatory tail generated by the singularity in the dielectric function, at the Fermi level. They are expected to be obtainable using the pseudopotential and electron screening theories. These potentials are typical of simple metallic liquids like the alkali metals and most of the polyvalent metals. For noble metals and transition metals of $3 \mathrm{~d}$ and $4 \mathrm{~d}$ series, there are serious impediment in formulating theoretical description because of the hybridization between the d-electrons and the s-electrons. The same is true for the rare earth liquids due to the strong f-electron binding. The pseudopotential method has been generalized by Harrison[44] to be applied to the transition and noble metals, with more or less success. There is another class of elements, which cannot readily be treated within the pseudopotential theory, like liquid Te that show evidence of chain like structure. They have high resistivity and do not fall into the class of simple liquids.

Unlike the pair potentials of the rare gases which are independent of the volume, those issued from pseudopotential are volume-dependent via the wave vector at the Fermi level $k_{F}$. It is clear from preceding discussions that the interionic potentials $u(r)$ in simple liquid metals depend on the dielectric function $\varepsilon\left(q, k_{F}\right)$, through the normalized energy wave number characteristic $F_{N}(q)$. The form of the interionic potential is very sensitive to the pseudopotental used and to the approximations in the electron screening. As a consequence, the pseudopotential is not a unique quantity. The Ashcroft model has proved of great value to bring out the basic ideas of the pseudopotential theory, but it sometimes fails because of its oversimplified form. The accuracy of any calculation of reliable interionic potential may be improved by using pseudopotentials spatially non-local, for which the form factor depends not only on $q$ but also on the wave vector $\mathbf{k}$.

Once the form factor $\left\langle\mathbf{k}+\mathbf{q}\left|w_{0}\right| \mathbf{k}\right\rangle$ and the dielectric function $\varepsilon\left(q, k_{F}\right)$ are known, the interionic potential may be calculated via Eq. (154), with the intention of determining a variety of structural and thermodynamic properties. To test an interionic potential $u(r)$, theories often attempt to link $u(r)$ to the structure factor $S(q)$ directly accessible to measurement by X-ray diffusion or neutron scattering. Another basic achievement of the pseudopotential theory has been to underline the proposition that the form factor $\left\langle\mathbf{k}+\mathbf{q}\left|w_{0}\right| \mathbf{k}\right\rangle$ and the dielectric function $\varepsilon\left(q, k_{F}\right)$ are capable of predicting the electron transport properties of pure liquid metals[45].

As already mentioned, the presence of the electron gas makes that the potential energy function consists of a sum of interionic potentials plus a volume-dependent term (whereas structure-independent) playing a dominant role in determining the thermodynamic properties, but quite difficult to evaluate properly. Thus, there is a number of liquids for which the use of pseudopotentials is wholly inappropriate, and problems still remain not fully resolved in the thermodynamics of metallic liquids, among them the metal-nonmetal transition in expanded liquid metals, especially along the liquid-vapor coexistence curve. Even for small expansion, failure will set in when the critical point is approached[19, 46, 47]. Furthermore, the transition across the interface from metallic behavior in the bulk liquid to insulating behavior in the coexisting vapor has been investigated[48, 49] by the theory of the surface tension of insulating liquids [50, 51].

Another problem of great interest is the transferability of a pseudopotential from the solid state to the liquid state with the same values of parameters. Of course, to increase our confidence in such a pseudopotential it is worthwhile to demonstrate how well many different physical properties of the liquid are predicted with it. Good results have been obtained with the Fiolhais et al.[52] structured pseudopotential, whose the parameters are designed for the solid state, specially for the alkali metals $[53,54]$. This pseudopotential is based on first principle arguments and does not represent an attempt to obtain agreement with experiment at all costs. The structure factor and isothermal compressibility of the divalent liquid metals are quite well reproduced[55]. Though reasonable results have been also obtained for the structural properties of the polyvalent metals, it appears that the melting point is largely overestimated[56].

Today the pseudopotential theory is supplanted by the density functional theory (DFT) established itself to compute the electronic structure in most branches of chemistry and materials science. In the formulation given by Kohn and coworkers $[57,58]$ the many-electron wave function is replaced by the electron density, so that the energy is just a functional of the latter. The DFT is applied, with low computational cost and reasonable accuracy, to predict diverse properties as binding or atomization energies, shapes and sizes of molecules, crystal structures of solids, energy barriers to various processes, etc. In the mid 1980s, it became an appreciable alternative to the well developed wave function techniques such as the HartreeFock one, when crucial developments in exchange-correlation energy have been taken into account[43].

DFT is a powerful tool to investigate the static properties of electronic systems. It is also a convenient tool to be used in conjunction with ab initio molecular dynamics (AIMD) simulations for extended systems[59]. Whereas classic MD describes trajectories of atoms as objects within an empirical interaction potential, the AIMD computations are simulations involving the motions of both nuclei and electrons. The evolution of the kinetic energies of both electrons and nuclei is observed in an identical way through the use of fictitious 
masses. Plane waves are usually used for the description of valence orbitals, whereas pseudopotential approximations are employed for taking into account the core electrons. The combination of quantum mechanics and molecular mechanics in AIMD is certainly one of the most promising theoretical tools available for theoretical chemistry. Its implementation for ab initio molecular dynamics simulation of large systems has also been successfully applied to solid state and liquid state physics, as well as to materials science.

\section{References}

[1] Philipps JC and Kleinman J (1959) New Method for Calculating Wave Functions in Crystals and Molecules. Phys Rev 116: 287-294.

[2] Born M, Mayer JE (1932) Zur Gittertheorie der Ionenkristalle. Z Physik 75: 1.

[3] Keesom WH (1921) Van der Waals attractive forces. Physik Z 22: 129.

[4] Hirschfelder JO, Curtiss CF, Bird RB (1954) Molecular Theory of Gases and Liquids, Wiley, New York, p. 245.

[5] London F (1937) The general theory of molecular forces. Trans Faraday Soc 33: 8-26.

[6] Israelachvili JN (1974) The nature of van der waals forces. Comtemp Phys 15: 159-177.

[7] Axilrod BM, Teller E (1943) Interaction of the van der Waals Type Between Three Atoms. J Chem Phys 11: 299-300.

[8] Axilrod BM (1951) Triple-Dipole Interaction: Theory. $J$ Chem Phys 19: 719-724.

[9] Doran MD, Zucker IJ (1971) Higher order multipole three-body van der Waals interactions and stability of rare gas solids. J Phys C: Solid Stat Phys 4: 307-312

[10] Bruch LW, McGee IJ (1973) Calculations and estimates of the ground state energy of helium trimers. J Chem Phys 59: 409-413.

[11] Bomont JM, Bretonnet JL, van der Hoef MA (2001) Comparison between integral equation method and molecular dynamics simulation for three-body forces: Application to supercritical argon. J Chem Phys 114: 5674-5681.

[12] Slater JC, Kirkwood JG (1931) The Van Der Waals Forces in Gases. Phys Rev 37: 682-697.

[13] Aziz RA, Slaman MJ (1986) The argon and krypton interatomic potentials revisited. Mol Phys 58: 679-697.
[14] Bretonnet JL (2011) Thermodynamic Perturbation Theory of Simple Liquids, Published in the book: Thermodynamics - Interaction Studies - Solids, Liquids and Gases, p. 839-870. https://www.intechopen.com/

[15] Hoover WG, Ree FH (1967) Use of Computer Experiments to Locate the Melting Transition and Calculate the Entropy in the Solid Phase. J Chem Phys 47: 4873-4878.

[16] Hoover WG, Ree FH (1968) Melting Transition and Communal Entropy for Hard Spheres. J Chem Phys 49: 3609-3617.

[17] Chacon E, Reinaldo-Falagan M, Velasco E, Tarazona $\mathrm{P}$ (2001) Layering at Free Liquid Surfaces. Phys Rev Lett 87: 166101-1-4 .

[18] Li D, Rice SA (2004) Some Properties of "Madrid" Liquids. J Phys Chem B 108: 19640-19646.

[19] Bomont JM, Bretonnet JL (2006) An effective pair potential for thermodynamics and structural properties of liquid mercury. J Chem Phys 124: 054504-1-8.

[20] Ashcroft NW (1966) Electron-ion pseudopotentials in metals. Phys Lett 23: 48-50.

[21] Heine V, Abarenkov IV (1964) A new method for the electronic structure of metals. Phil Mag 9: 451-465.

[22] Abarenkov IV, Heine V (1965) The model potential for positive ions. Phil Mag 12: 529-537.

[23] Shaw RW, Harrison WA (1967) Reformulation of the Screened Heine-Abarenkov Model Potential. Phys Rev 163: 604-611.

[24] Heine V (1970) The Pseudopotential Concept. Solid State Phys 24: 1-36.

[25] Harrison WA (1966) Pseudopotentiels in the Theory of Metals, Benjamin, New York, p. 46.

[26] Shaw RW (1968) Optimum Form of a Modified HeineAbarenkov Model Potential for the Theory of Simple Metals. Phys Rev 174: 769-781.

[27] Shaw RW (1969) Application of the optimized model potential to calculation of energy-wave-number characteristics for simple metals. J Phys C: Solid State Phys 2: 2335-2349.

[28] Hubbard J (1957) The Description of Collective Motions in Terms of Many-Body Perturbation Theory. II. The Correlation Energy of a Free-Electron Gas. Proc Roy Soc (London) A 243: 336-352.

[29] Lindhard J (1954) On the properties of a gas of charged particles. Kgl Danske Videnskab Selskab Mat Fys Medd 28: 8 . 
[30] Friedel J (1952) The distribution of electrons round impurities in monovalent metals. Phil Mag 43: 153-189.

[31] Shimoji M (1977) Liquid Metals, Acad. Press London, p. 105.

[32] Hafner J (1987) From Hamiltonians to Phase Diagrams, Springer-Verlag, Berlin .

[33] Young WH (1992) Structural and thermodynamic properties of NFE liquid metals and binary alloys. Rep Prog Phys 55: 1769-1853.

[34] Pines D, Nozires P (1966) The Theory of Quantum Liquids 1, Benjamin, New York.

[35] Raimes S (1967) The Wave Mechanics of Electrons in metals, North-Holland Publishing. Company, Amsterdam.

[36] Ashcroft NW, Stroud D (1978) Theory of the Thermodynamics of Simple Liquid Metals. Solid State Physics 33: 1-81.

[37] Baus M, Hansen JP (1980) Statistical mechanics of simple coulomb systems. Physics Reports 59: 1-94.

[38] Ichimaru S (1982) Strongly coupled plasmas: highdensity classical plasmas and degenerate electron liquids. Rev Mod Phys 54: 1017.

[39] March NH, Tosi MP (1984) Coulomb Liquids, Acad Press, London, p. 70.

[40] Vashishta P, Singwi KS (1972) Electron Correlations at Metallic Densities. Phys Rev B 6: 875-887.

[41] Ichimaru S, Utsumi K (1981) Analytic expression for the dielectric screening function of strongly coupled electron liquids at metallic and lower densities. Phys Rev B 24: 7385-7388.

[42] Farid B, Heine V, Engel GE, Robertson IJ (1993) Extremal properties of the Harris-Foulkes functional and an improved screening calculation for the electron gas. Phys Rev B 48: 11602-11621.

[43] Bretonnet JL (2017) Basics of the density functional theory, AIMS Materials Science, 4 (6): 1372-1405. http://www.aimspress.com/journal/Materials

[44] Harrison WA (1969) Transition-Metal Pseudopotentials. Phys Rev 181: 1036-1053.

[45] Ziman JM (1967) The electron transport properties of pure liquid metals. Advan Phys 16: 551-580.

[46] Hensel F (1990) Critical behaviour of metallic liquids. $J$ Phys: Condens Matter 2: SA33-SA45.
[47] Bomont JM, Delhommelle J, Bretonnet JL (2007) Structure and thermodynamics of the expanded liquid mercury by Monte Carlo simulation: a first attempt. $J$. Non-Cryst. Solids 353: 3454-3458.

[48] Bomont JM, Bretonnet JL (2008) A molecular dynamics study of density profiles at the free surface of liquid mercury. J Phys: Conf Ser 98: 042018-1-5.

[49] Bomont JM, Bretonnet JL, Gonzalez DJ, Gonzalez LE (2009) Computer simulation calculations of the free liquid surface of mercury. Phys. Rev. B 79: 144202-14.

[50] Evans R, Kumaravadivel R (1976) A thermodynamic perturbation theory for the surface tension and ion density profile of a liquid metal. J Phys C: Solid State Phys 9: 1891-1906.

[51] D'Evelyn MP, Rice SA (1983) A study of the liquidvapor interface of mercury: Computer simulation results. J Chem Phys 78: 5081-5095.

[52] Fiolhais C, Perdew JP, Armster SQ, McLaren JM, Brajczewska M (1995) Dominant density parameters and local pseudopotentials for simple metals. Phys. Rev. B 51: 14001-14011. (1996) Erratum: Dominant density parameters and local pseudopotentials for simple metals. Phys Rev B 53, 13193.

[53] Boulahbak M, Jakse N, Wax JF, Bretonnet JL (1998) Transferable pair potentials for the description of liquid alkali metals. J Chem Phys 108: 2111-2116.

[54] Wax JF, Albaki R, Bretonnet JL (2001) Temperature dependence of the diffusion coefficient in liquid alkali metals. Phys Rev B 65: 014301-1-9.

[55] Wax JF, Albaki R, Bretonnet JL (2000) Structural and dynamique properties of liquid alkali-earth metals near the melting point. Phys Rev B 62: 14818-14827.

[56] Mendoub EB, Albaki R, Charpentier I, Bretonnet JL, Wax JF, Jakse N. (2007) Molecular dynamics and integral equation study of the structure and thermodynamics of polyvalent liquid metals. J. Non-Cryst. Solids 353: 3475-3479.

[57] Hohenberg P, Kohn W (1964) Inhomogeneous Electron Gas. Phys Rev 136: B864-B871.

[58] Kohn W, Sham LJ (1965) Self-Consistent Equations Including Exchange and Correlation Effects. Phys Rev 140: A1133-A1138.

[59] Car R, Parrinello M (1985) Unified Approach for Molecular Dynamics and Density-Functional Theory. Phys Rev Lett 55: 2471-2474. 